
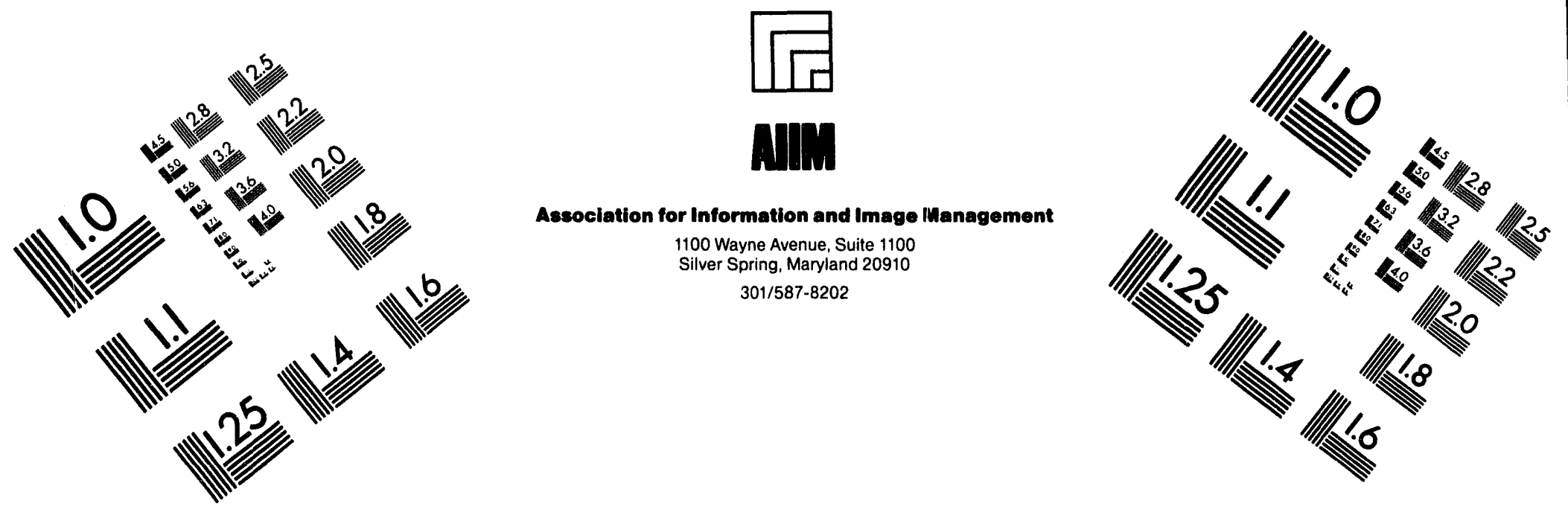

Centimeter

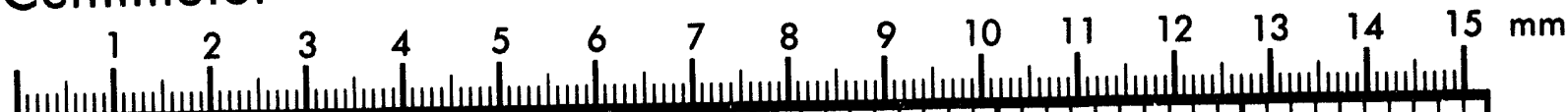

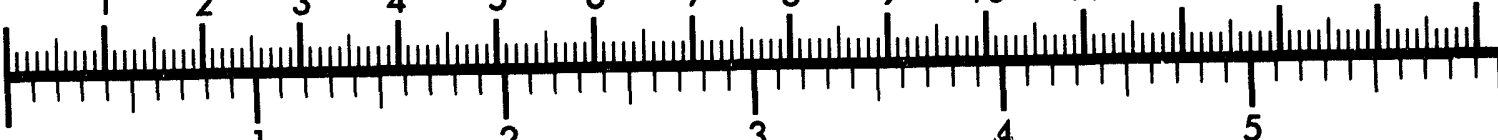
Inches
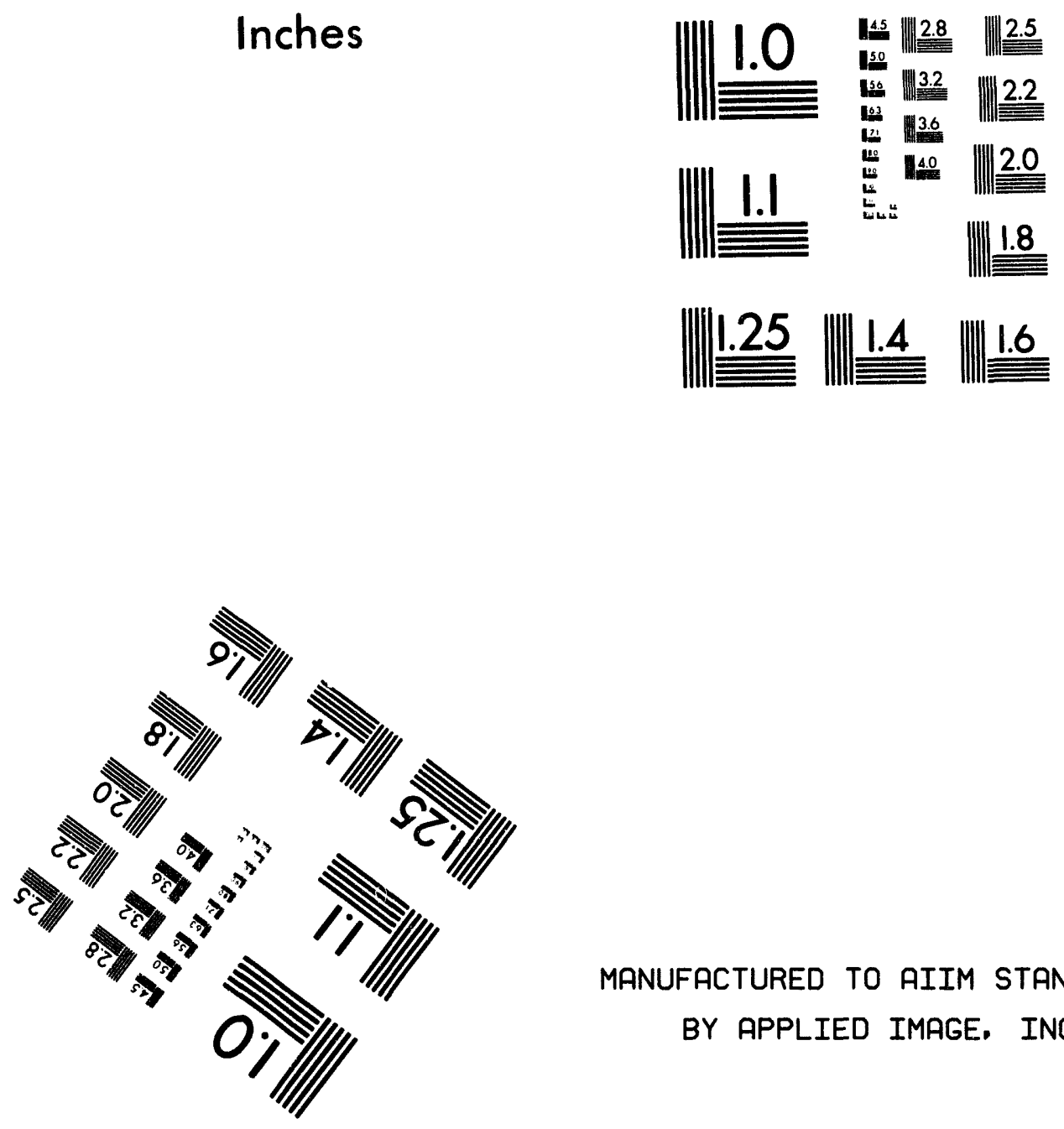

MANUFACTURED TO AIIM STANDARDS BY APPLIED IMAGE, INC.

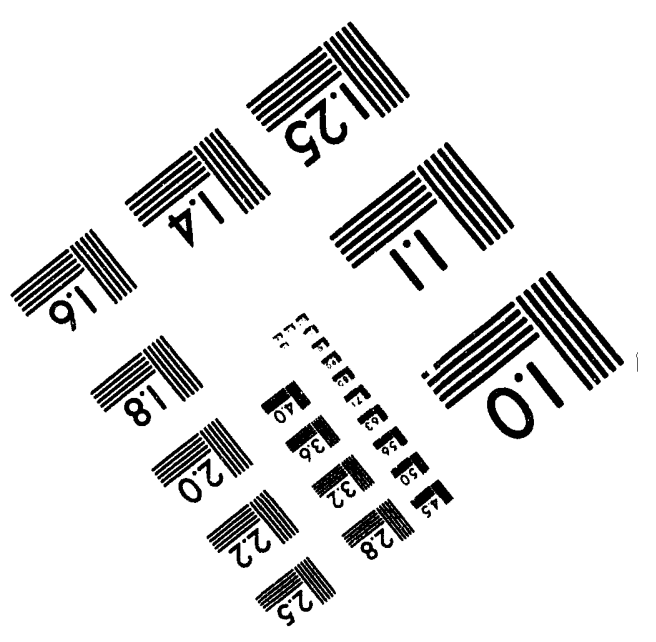



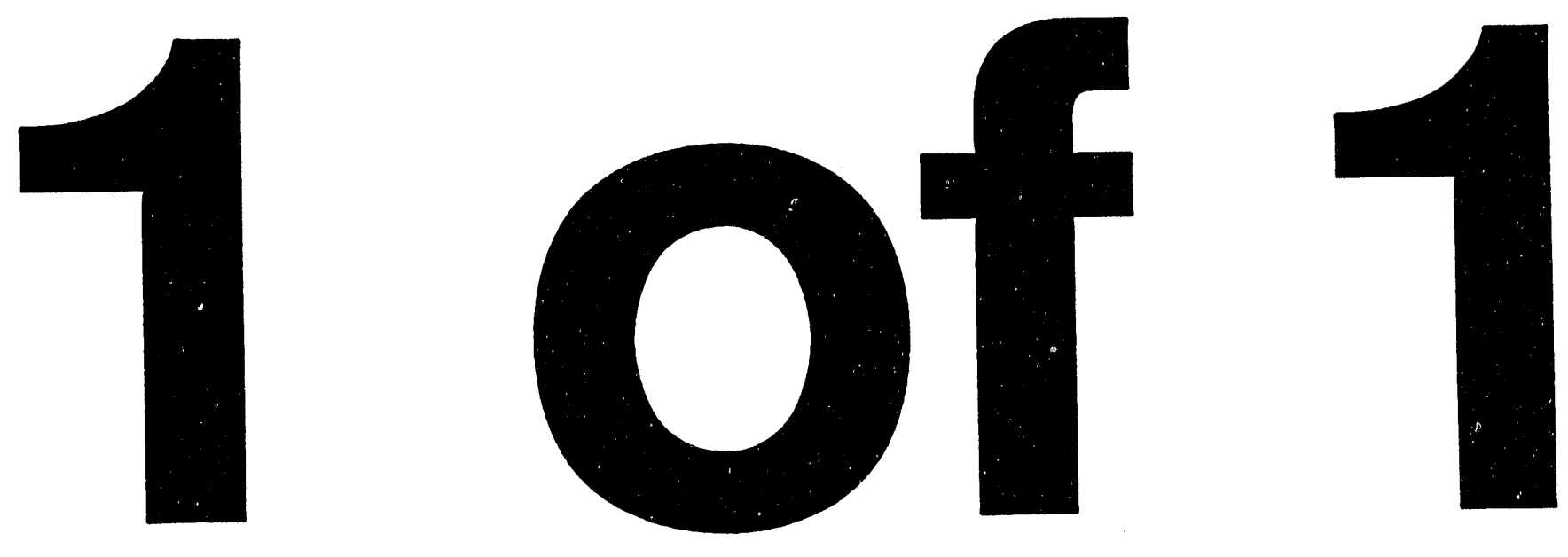


\section{Predictive Modeling of Particle-Laden Turbulent Flows}

\section{FINAL REPORT}

to the Department of Energy Pittsburgh Energy Technology Center Pittsburgh, PA

Project Manager: Franklin Shaffer
from the Department of Chemical Engineering - Carnegie Mellon University Pittsburgh, Pennsylvania 15213

Graduate Students: E.J. Bolio and C.M. Hrenya

Jennifer L. Sinclair

Grant Number: DE-FG22-92PC92540 


\begin{abstract}
Earlier work of Sinclair and Jackson which treats the laminar flow of gas-solid suspensions is extended to model dilute turbulent flow. The random particle motion, often exceeding the turbulent fluctuations in the gas, is obtained using a model based on the kinetic theory of granular materials. A two-equation low Reynolds number turbulence model is modified to account for the presence of the dilute particle phase. Comparisons of the model predictions with available experimental data for the mean and fluctuating velocity profiles for both phases indicate that the resulting theory captures many of the flow features observed in the pneumatic transport of large particles. The model predictions did not manifest an extreme sensitivity to the degree of inelasticity in the particle-particle collisions for the range of solid loading ratios investigated.
\end{abstract}




\section{Introduction}

Dispersed gas-sc lid turbulent flows are encountered in a wide variety of chemical processes. Such processes include coal injection into entrained-flow gasifiers, pneumatic conveying of solids in transport lines, and high velocity fluidization as found in circulating fluidized beds (CFBs). These systems are often characterized by complex flow phenomena such as nonuniform spatial distribution of particles, large slip velocities between the two phases, suppression or enhancement of the gas-phase turbulent intensity, and the existence of several possible pressure gradients and solids holdup for specified values of gas and solids flow rates. An accurate understanding of the turbulent flow behavior of gas-solid mixtures is necessary for scaleup, design and optimization of such processes.

In order for a mathematical model to accurately predict all of the flow phenomena associated with gas-solid flows, each of the mechanical interactions present in the system must be included in the model. These interactions, as outlined in Sinclair and Jackson (1989), which depend on the mean and fluctuating components of the gas and solid velocity fields, include the following:

1. The interaction between the mean gas velocity and mean solid velocity which gives rise to the drag force between the two phases.

2. The interaction between the mean and fluctuating gas velocities which generates the gasphase Reynolds stresses.

3. The interaction between the mean and fluctuating solid velocities which generates stresses in the particle assembly.

4. The interaction between the particles and the turbulent fluctuations of gas velocity which results in a flux of kinetic energy which either damps the fluctuations in the gas phase and stimulates fluctuations in the particle phase, or vice versa.

Recent models (Sinclair and Jackson, 1989; Ding and Gidaspow, 1990; Pita and Sundaresan, 1991; Pita and Sundaresan, 1993; Ocone et al., 1993) have been able to qualitatively predict many of the flow phenomena associated with particle-laden flows by incorporating only the 
first and third effects mentioned above. For the large particles considered in these studies, stresses in the solid phase are described by making an analogy between the random particle motion due to particle collisions and the thermal motion of molecules in a gas, and employing methods from kinetic theory. Momentum transfer in the solid-phase occurs both by translation of particles between adjacent layers and by brief particle-particle contacts. The quantitative ability of these models is uncertain, however, since they do not include descriptions for the interactions which involve gas-phase fluctuations.

Louge et al. (1991) were the first to expand on the above models to include the effects of both gas-phase turbulence and particle collisions for systems of low particle concentrations. Similar to the Sinclair and Jackson model, solid phase stresses are described using the kinetic theory analogy. Gas-phase turbulent stresses are modelled via a high Reynolds one-equation closure which requires a pre-specified mixing length. Since this closure scheme is not valid in the viscous sublayer, wall functions are used to determine the velocity distribution in these regions. The model also contains a modified form of the expression proposed by Koch (1990) to approximate the correlation between the velocity fluctuations of the gas and particles in dilute-phase flows. Model predictions for steady, fully-developed vertical pipe flow were compared with the experimental measurements of Tsuji et al. (1984). Although the predictions of both phases were in good agreement with the data at the lower loadings investigated by Tsuji et al., the predictive ability of the model worsened at the higher loadings. Specifically, the model was unable to predict the extreme flattening of the mean and fluctuating gas velocity profile which was observed experimentally. This inability of the model to give good predictions at higher loadings is not surprising, however, since the expressions used for the mixing length and wall functions are based on single phase, clear gas flow. Even for dilute phase flows, the universality of such functions begins to break down as the concentration of particles increases (Rizk and Elghobashi, 1989).

Another class of two phase models which account for velocity fluctuations in the gas and solid phase was proposed primarily by researchers in the mechanical engineering community, namely Elghobashi and Abou-Arab (1983), Pourahmadi and Humphrey (1983), Chen and Wood 
(1985), and Rizk and Elghobashi (1989). All of these models neglect particle-particle interactions and consider systems with particles small enough such that they follow the fluid motion closely. (For example, the model of Chen and Wood is restricted to particles with diameters $-<100 \mu \mathrm{m}$.) Solid-phase stresses are described empirically as fractions of the corresponding gas-phase stresses; thus, these expressions are not valid for systems composed of larger particles in which the particle velocity fluctuations often exceed those of the gas.

In this publication we seek to expand on the previous effort of Louge et al. (1991) by incorporating a two-equation, low Reynolds number $k-\varepsilon$ closure to describe gas-phase stresses. This closure model, which is extended to account for the presence of a dilute particle phase, is formally integrable up to the pipe wall, and thereby the need for empirical wall functions is eliminated. Stresses in the particle phase and the boundary conditions for the particle phase follow the analysis of Sinclair and Jackson (1989). The dilute turbulent gas-solid flow model presented here considers particle velocity fluctuations at the level of individual particles only, in contrast to a recent model given by Dasgupta et al. (1993) which describes the organized motion of groups of particles using a speculative $k-\varepsilon$ closure model for the particle phase. The model outlined in this work captures most of the flow features observed in the dilute, steady, fully-developed vertical pipe flow measurements of Tsuji et al. (1984), Lee and Durst (1982), and Maeda et al. (1980) obtained using laser Doppler velocimetry. In addition, excellent agreement is obtained between previously unpublished experimental data (Tsuji, 1993) for solid velocity fluctuations (which exceed the gas velocity fluctuations) and our model predictions. We show that simpler dilute turbulent gas-solid flow models such as those which neglect the effect of gas-phase turbulence or particle-particle interactions can not describe the flow features observed in the experimental data. The model predictions are also not extremely sensitive to the value of the coefficient of restitution for the range of solids loadings (solid-to-gas mass flow rate ratio) investigated here. 


\section{Mathematical Model}

The governing equations for the flow of a fluid-solid mixture were developed using a continuum approach which replaces the point mechanical variables, such as velocities and pressures, with their corresponding local mean variables (Anderson and Jackson, 1967).

\section{Governing Equations - Gas Phase}

For steady, turbulent, fully-developed gas-flow in a vertical pipe, a time smoothing of the axial component of the gas momentum balance yields

$$
0=-\frac{\partial p}{\partial z}-\beta\left(v_{z}-u_{z}\right)+\frac{1}{r} \partial r\left(r \frac{\partial T_{r z}}{\partial r}\right)
$$

where

$$
\tau_{r z}^{T}=\mu \frac{\partial v_{z}}{\partial r}-\rho \overline{v_{r}^{\prime} v_{z}^{\prime}}
$$

Here $\mathrm{v}$ refers to the gas phase velocity, $\beta$ is the drag coefficient which depends upon the local slip velocity and solid volume fraction $v$, and $\tau^{T}$ is the total stress tensor for the gas phase. We adopt a closure of the Reynolds stress by assuming that the stress is related to the mean rate of strain through the eddy viscosity $\mu_{\mathrm{t}}$.

$$
-\rho \overline{v_{r}^{\prime} v_{z}^{\prime}}=\mu_{t} \frac{\partial v_{z}}{\partial r}
$$

It is also assumed that the eddy viscosity is uniquely determined by $k$,the turbulent kinetic energy of the gas, where

$$
k=\frac{1}{2} \frac{v_{i}^{\prime} v_{i}^{\prime}}{1}
$$

and $\varepsilon$, the rate of dissipation of $\mathbf{k}$, via the Kolmogorov-Prandtl relation

$$
\mu_{\mathrm{t}}=\frac{\mathrm{c}_{\mu} \mathrm{f}_{\mu} \rho_{\mathrm{g}} \mathrm{k}^{2}}{\varepsilon}
$$

where $f_{\mu}$ is a function of the turbulent Reynolds number $R_{T}$. A form for the drag coefficient is based on an expression suggested by Ding and Gidaspow (1990).

$$
\beta=\frac{3}{4} \frac{\rho_{g}}{d} C_{D} \frac{v}{(1-v)^{2.65}}\left|v_{z}-u_{z}\right|
$$

where 


$$
C_{D}=\frac{24}{\operatorname{Re}_{d}}\left(1+0.15 \operatorname{Re}_{d}^{0.687}\right)
$$

In single phase flows $(v=0)$ the intensity of the turbulent fluctuations can be found from the following transport equation for $\mathrm{k}$.

$$
0=\frac{1}{r \partial r}\left|r\left(\mu+\frac{\mu_{t}}{\sigma_{k}}\right) \frac{\partial k}{\partial r}\right|+\mu_{t}\left(\frac{\partial v_{z}}{\partial r}\right)^{2}-\rho_{g} \varepsilon
$$

The terms in this equation describe the balance between the diffusive transport by turbulent motion, the production by interaction of turbulent stresses and mean velocity gradients, and the destruction by dissipation, respectively. In one-equation closure models, the dissipation rate is determined by prespecified turbulent mixing length relations. However, in two-equation models the dissipation rate is given by the solution of a separate transport equation.

$$
0=\frac{1}{r} \partial \mathrm{r}\left|\mathrm{r}\left(\mu+\frac{\mu_{\mathrm{t}}}{\sigma_{\varepsilon}}\right) \frac{\partial \varepsilon}{\partial \mathrm{r}}\right|+\mathrm{c}_{1} \mathrm{f}_{1} \frac{\varepsilon}{\mathrm{k}} \mu_{\mathrm{t}}\left(\frac{\partial \mathrm{v}}{\partial \mathrm{r}}\right)^{2}-c_{2} \mathrm{f}_{2} \rho_{\mathrm{g}} \frac{\varepsilon^{2}}{\mathrm{k}}
$$

The terms in the above equation represent physical processes similar to those discussed already for the $k$ equation; that is, the equation represents a balance of diffusive transport, production, and destruction of $\varepsilon$.

A low Reynolds number version of the standard $k-\varepsilon$ closure model is used as these closure models allow integration up to the pipe wall by employing functions $f_{1}, f_{2}$, and $f_{\mu}$ which reproduce the behavior of $k$ and $\varepsilon$ near the wall (Table 1). The particular base two-equation closure model given here is the low-Reynolds number k- $\varepsilon$ Myong and Kasagi (1990) model. Our choice of the Myong and Kasagi version of the low Reynolds k- $\varepsilon$ closure is based on a comparative study (Hrenya et al., 1993). The Myong and Kasagi model gives the best predictions for single-phase turbulent pipe flow and is the only model to yield the correct qualitative shape of the eddy viscosity profile. One disadvantage of $k-\varepsilon$ closure models, however, is that they neglect the anisotropy of the velocity fluctuations. Although more sophisticated turbulence closures which do account for the anisotropic nature of turbulence are available, recent studies by Martinuzzi and Pollard (1989) indicate that the predictions from a low-Reynolds $k-\varepsilon$ closure are in better agreement with 
experimental pipe flow data than the predictions from algebraic stress and Reynolds stress closure models.

For two-phase flows, the gas phase turbulent kinetic energy equation and its dissipation rate equation must be modified to account for the presence of a dilute particle phase. By making similar order of magnitude arguments as Louge et al. (1991), the gas-phase turbulent kinetic energy equation becomes

$$
\begin{gathered}
0=\frac{1}{r} \frac{\partial}{\partial r}\left\{r(1-v)\left(\mu+\frac{\mu_{t}}{\sigma_{k}}\right) \frac{\partial k}{\partial r}\right\}+(1-v) \mu_{t}\left(\frac{\partial v_{z}}{\partial r}\right)^{2} \\
-\rho_{g} \varepsilon(1-v)-\beta(1-v)\left(2 k-\overline{v_{i}^{\prime} u_{i}^{\prime}}\right)
\end{gathered}
$$

where $u$ is the particle velocity. The last term in the turbulent kinetic energy equation accounts for turbulence dissipation and generation due to the interaction between the phases. The equation for the rate of dissipation of gas phase turbulent kinetic energy is given correspondingly as

$$
\begin{aligned}
0=\frac{1}{r} \partial r & \left|r(1-v)\left(\mu+\frac{\mu_{t}}{\sigma_{\varepsilon}}\right) \frac{\partial \varepsilon}{\partial r}\right|+c_{1} f_{1} \frac{\varepsilon}{k}(1-v) \mu_{t}\left(\frac{\partial v_{2}}{\partial r}\right)^{2} \\
& -c_{2} f_{2} \rho_{g}(1-v) \frac{\varepsilon^{2}}{k}-c_{3} \beta(1-v)\left(2 k-\overline{v_{i}^{\prime} u_{i}^{\prime}}\right) \frac{\varepsilon}{k}
\end{aligned}
$$

As a tentative first attempt at closure, the correlation $\overline{v_{i}^{\prime} u_{i}^{\prime}}$ is modelled as

$$
\overline{v_{i}^{\prime} u_{i}^{\prime}}=\frac{4}{\sqrt{\pi}} \frac{d}{\rho_{s}} \beta \frac{(1-v)}{v} \frac{\left(v_{z}-u_{z}\right)^{2}}{\sqrt{T}}
$$

following Louge et al. (1991), where $\mathrm{T}$ is a "particle temperature" that is a measure of the

magnitude of the particle velocity fluctuations $\left[T=\frac{1}{3}\left(\overline{u^{\prime} 2}\right)\right]$. The closure is based on an expression developed by Koch (1990) who considers the case of a dilute suspension of particles where the particle velocity distribution function is controlled by solid-body interactions. In this paper we evaluate this closure and indicate its shortcomings. Work is in progress to improve the description for how the particles affect the gas turbulence incorporating a treatment developed by Yuan and Michaelides (1992).

\section{Goveming Equations - Particle Phase}

Axial and radial force balances for the particle phase take the following form 


$$
\begin{aligned}
& 0=-\frac{1}{r} \frac{\partial}{\partial r}\left(r \frac{\partial \sigma_{r z}}{\partial r}\right)+\beta\left(v_{z}-u_{z}\right)-\rho_{s} v g \\
& 0=\frac{1}{r} \frac{\partial}{\partial r}\left(r \frac{\partial \sigma_{r}}{\partial r}\right)-\frac{\sigma_{\theta \theta}}{r}
\end{aligned}
$$

The particles are assumed to be spherical, cohesionless and uniform in size. Stresses in the particle assembly resulting from translation of particles between adjacent layers and by particle collisions are denoted by the stress tensor $\underline{\underline{\sigma}}$, defined in the compressive sense. Since the particle-phase effective pressure and viscosity depend strongly on the magnitude of the particle velocity fluctuations, these must be found by solving a separate differential equation representing a balance for the kinetic energy associated with the random particle motion or "pseudo-thermal energy".

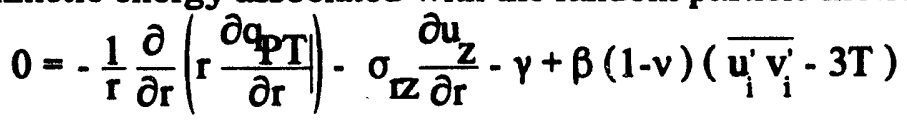

Pseudo-thermal energy is conducted as a result of gradients in the particle temperature, generated by shear, and dissipated by the inelasticity of collisions between particles. The last term represents a source and sink of the energy for the particles' fluctuating motion due to fluid-particle interactions. In order to close these equations, the granular kinetic theory constitutive relations of Lun et al. (1984) with a few modifications (detailed in Sinclair and Jackson, 1989) are used for the particle phase stress tensor $\underline{\underline{\sigma}}$, the pseudo thermal energy flux vector $\underline{q}_{\mathrm{PT}}$, and the dissipation rate of pseudo-thermal energy $\gamma$ (Table 2).

\section{Boundary Conditions}

In summary, the dilute particle-laden turbulent flow model is comprised of five coupled differential equations (Eqns. 1, 10, 11, 13, and 15) and one algebraic equation (Eqn. 14) which describe the relationship between the mean and fluctuating components of the gas and solid velocities $\left(v_{z}, u_{z}, k, T\right)$, the dissipation rate of gas phase turbulent kinetic energy $\varepsilon$, the solids volume fraction $v$, and the pressure gradient. The radial momentum balance (Eq. 14) simplifies to an algebraic relationship between the particle temperature and the solids volume fraction. A complete formulation of the model requires boundary conditions at the tube wall and centerline. At 
the axis of the pipe, symmetry clearly demands that the gradients of all the variables be zero. The mean gas velocity and gas turbulent kinetic energy are zero at the wall. The wall boundary condition for the dissipation rate $\varepsilon$ is obtained by a reduction of the $k$ equation near the wall which yields

$$
\varepsilon=\frac{\mu}{\rho_{g}} \frac{\partial 2 k}{\partial r 2}
$$

As a first attempt for this boundary condition, we have chosen to neglect the additional fluidparticle interaction terms appearing in the $k$ equation.

In general it is not permissible to set the particle velocity and the pseudo-thermal temperature equal to zero at a solid wall. Exceptions occur when the wall is sufficiently rough, minimizing particle slip, and when the bounding wall is sufficiently "soft", creating highly inelastic particle-wall collisions. Following Johnson and Jackson (1987), a boundary condition for the particle velocity at the wall is found by equating the momentum flux transmitted to the wall by virtue of particle-wall collisions to the stress in the particle assembly adjacent to the wall.

$$
\frac{\rho_{s} \pi u_{2} \phi \sqrt{T}}{2 \sqrt{3}\left(\frac{v_{0}}{v}-\frac{v_{0}^{2 / 3}}{v^{2 / 3}}\right)}=\sigma_{r 2}
$$

The transfer rate of axial momentum at the wall depends on the fraction of diffuse particle-wall collisions given by a specularity factor $\phi$, which varies between zero and one. Similarly, a boundary condition for the pseudo-thermal temperature at the wall is found by equating the energy conducted to the wall by virtue of particle-particle collisions to the energy lost at the wall by particle-wall collisions and the energy generated by specular particle-wall collisions.

$$
\mathrm{q}_{\mathrm{PT}_{\mathrm{r}}}=\frac{\sqrt{3} \rho_{\mathrm{s}} \pi\left(1-\mathrm{e}_{\mathrm{w}}^{2}\right) \mathrm{T}^{3 / 2}}{4\left(\frac{v_{0}}{v}-\frac{v_{0}^{2 / 3}}{v^{2 / 3}}\right)} \cdot \frac{\rho_{\mathrm{s}} \pi u_{\mathrm{z}} 2 \phi / T}{2 \sqrt{3}\left(\frac{v_{0}}{v}-\frac{v_{0}^{2 / 3}}{v^{2 / 3}}\right)}
$$




\section{Results and Discussion}

The governing equations together with their boundary conditions were solved using an adaptation of the implicit finite-difference marching technique developed by Patankar and Spalding (1967). In this method, the problem is formulated as a pseudo-transient one and integrated in time until the steady state solution is obtained. Sixty radial grid points were placed nonuniformly throughout the domain with a concentration of points near the wall and tube axis, and the computed profiles vere verified by checking the sensitivity of the solutions to an increase in the number of grid points. For the range of solid loadings investigated here, in which the solids are distributed rather uniformly over the tube cross-section, the model solutions represent unique flow predictions. At higher solids loadings, however, in which significant segregation of solids are observed, more than one operating condition and model solution are possible for a given solids flux and superficial gas velocity (Sinclair and Jackson, 1989; Pita and Sundaresan, 1991).

Figure 1 shows a validation of the base turbulence model; that is, the system of equations is first solved for the case of clear gas $(v=0)$ and compared with the experimental data of Schildknecht et al. , 1979). In these single-phase plots the frictional velocity is matched with the measured value by adjusting the input pressure gradient. Agreement between model prediction and experimental data is excellent at the level of the total wrbulent kinetic energy $\mathbf{k}$, but the predictions, while still good at the tube axis, fall below the data near the wall when comparisons are made at the level of the axial gas velocity fluctuations. This discrepancy is due to the isotropic turbulence assumption inherent in the $k-\varepsilon$ closure model. As previously mentioned, higher order closure models, which potentially could correct this inherent discrepancy, are not adopted here based on the recent study by Martinuzzi and Pollard (1989). Since laser Doppler measurements for twophase flows in vertical risers to date only report the magnitude of the axial gas velocity fluctuations, the type of discrepancy illustrated in Figure $1 \mathrm{~b}$ will be present when comparing model predictions employing the isotropic assumption with experimental data at the level of the axial 
fluctiations. This point is important in order to gauge if a model is accurately predicting the degree of turbulence modulation in the gas phase.

For two-phase flow, we compare the model predictions with available experimental measurements for steady, fully-developed flow in a vertical pipe. Maeda et al. (1980), Lee and Durst (1982), and Tsuji et al. (1984) report data for gas and solid velocity profiles using laser Doppler velocimetry. In order to generate model solutions for two-phase flow which match the reported operating conditions given the set of system parameters, we input a pressure gradient and solids volume fraction at the centerline and iterate on these two quantities until the specified solids loading and Reynolds number specified are obtained. Since Maeda et al. and Lee and Durst transported glass particles $\left(\rho_{\mathrm{s}}=2590 \mathrm{~kg} / \mathrm{m}^{3}\right.$ ) in a glass pipe (ID $56 \mathrm{~mm}$ and $41.8 \mathrm{~mm}$, respectively), the coefficients of restitution for particle-particle and particle-wall collisions are both assigned a value of 0.94 (Lun and Savage, 1986). In Tsuji et al's system polystyrene spheres $\left(p_{s}=1020 \mathrm{~kg} / \mathrm{m}^{3}\right)$ were conveyed in a glass pipe of $30.5 \mathrm{~mm}$ diameter, and values of 0.9 and 0.7 are assigned to $\mathrm{e}$ and $\mathrm{ew}_{\mathrm{w}}$, respectively. Since no independent measurements are available for the specularity factor, we chose one set of experimental measurements and varied this coefficient in our model until our predicted particle slip matched the measured slip. A value for $\phi$ equal to 0.002 was obtained by this procedure and retained in all of the comparisons at the various operating conditions, as the three sets of experimental measurements were made in glass pipes with approximately the same degree of wall roughness. The flat solid velocity profiles near the wall in the experimental measurements indicate that the fraction of tangentiai momentum lost is very small, and a small fraction of diffuse particle-wall collisions is reasonable. It should be emphasized that this coefficient can be obtained from detailed measurements of normal and tangential velocity changes when particles impact surfaces at varying incident angles (Hui et al., 1984). Measurement of these coefficients is the subject of our ongoing experimental work (Shaffer et al., 1994). 


\section{Mean Velocity Profiles'}

Figures 2, 3, and 4 present comparisons of the predicted mean gas and solid velocity profiles with the experimental measurements of Lee and Durst. The model captures the progressive increase in the particle slip with increasing particle size at similar operating conditions (solids loading and Reynolds number based on the tube diameter and centerline gas velocity). The

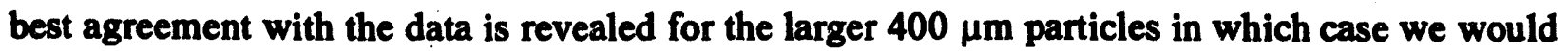
expect collective effects due to interactions between particles to be more significant. The dashed line indicates the mean solid velocity predictions from a model which neglects stresses in the particle phase due to particle-particle interactions. This simplified model predicts negative solid velocities at the tube wall. The present model predictions and the experimental data indicate a change in sign of the relative velocity near the wall.

Figures 5 and 6 compare model predictions for the mean gas and solid velocity profiles with the experimental data of Maeda et al. Figure 5 indicates that for small $45 \mu \mathrm{m}$ glass particles at very low solid loadings a simplified model which neglects particle phase stress is able to adequately predict the local flow structure. For these smaller particles the gas may act as a buffer minimizing direct solid-body contacts. In contrast, Figure 6 reveals that for the larger $136 \mu \mathrm{m}$ glass particles studied by Maeda et al., this same simplified model fails in a manner similar to that shown in the Lee and Durst comparisons even at very low solid loadings. The line with solid squares reveals the predictions from a highly simplified analysis which neglects both stresses in the solid phase and gas phase turbulence. As expected, the use of such a highly simplified model results in predictions which show very poor agreement with the experimental data for the gas and solid velocities.

In Figures 7 and 8 the mean velocity predictions for both phases are shown together with the experimental data for $200 \mu \mathrm{m}$ and $500 \mu \mathrm{m}$ polystyrene spheres at the lowest solids loadings investigated by Tsuji et al. Again, improved agreement between model predictions and experimental measurements is seen with the larger particles. Our model predictions closely 
resemble the results shown by Louge et al. (1991) at these lower solid loadings. In their paper they present predictions from a treatment which neglects particle shear stress which are not repeated here. For completeness, we have computed solutions to another simplified model, indicated by the dashed lines, which retains stresses in the particle phase but neglects Reynolds stresses in the gas phase. For the flow conditions shown in Figures 7 and 8 and the other conditions investigated here, the shape of the mean gas velocity profiles is insensitive to variations in the operating conditions with this simplified model. In general, the mean solid velocity profiles are not largely influenced by neglecting gas phase turbulence.

The measurements of Tsuji et al indicate that the addition of solid particles flattens the gas velocity profile in the core of the pipe and steepens it in the region near the wall. This effect is augmented with increased solids loading. In addition, for higher solids loading ratios they observed that the maximum in the gas velocity profile deviates from the tube centerline. The data in Figures 7 and 9 show that because the shape of the mean velocity profile is not altered significantly with increased solids loading, the radial location at which the relative velocity vanishes moves closer to the wall at higher solids loading ratios. All of these flow features are captured with the present model. The predictions for the particle slip at the wall may indicate the need for an improved value of the specularity factor. Any discrepancy between measurement and prediction of the wall slip may also be due to the manner in which the closure relations developed by Lun et al. were modified for low particle concentrations. For unconfined, dilute flows the kinetic or translational contribution to the particle phase stress tensor increases in an unbounded fashion as the mean free path between collisions increases. For confined flows, we damp the kinetic contribution to the stress as the mean free path between collisions approaches the pipe diameter so that the stress vanishes as the solid volume fraction tends to zero. We have employed the corrections adopted by Sinclair and Jackson and Louge et al. (1991) with a negligible difference in model predictions. The dashed line in Figure 9 indicates the predictions from a model which neglects the kinetic contribution to the particle phase stress and considers the collisional 
contribution only. It is clear that some fraction of the kinetic contribution should be retained in the low particle limit but the correct modification remains uncertain.

\section{Gas Velocity Fluctuation Profiles}

Figure 10 shows the predicted axial gas fluctuations compared with the experimental data for $200 \mu \mathrm{m}$ polystyrene spheres at the lowest and highest solids loadings investigated by Tsuji et al. The dashed line indicates the corresponding clear gas profile at the same Reynolds number. Note that the clear gas prediction at the lowest solids loading does not signify good agreement with the data for the reasons previously mentioned. In this case the degree of gas turbulence modulation in the presence of particles is merely on the order of the inherent discrepancy in the comparisons made at the level of the axial fluctuating component. The model predictions capture the extreme flattening of the gas velocity fluctuations at the higher solids loading. This marked change in shape of the $\mathrm{k}$ profile was not seen in the predictions of Louge et al. However, at both low and high solids loadings the model overestimates the damping of the gas velocity fluctuations in the presence of the solid particles. For the $500 \mu \mathrm{m}$ particles (not shown) the model predictions show damping of the gas velocity fluctuations while the experimental results indicate an enhancement in the gas turbulent fluctuations. With the present model, the magnitude of the $\mathrm{k}$ profile is largely governed by changes in the production term in the $k$ equation resulting from variations in the mean gas velocity profile. The decrease in the production of $\mathrm{k}$ which coincides with the flattening of the mean gas velocity profile, should be offset by a source of fluctuations perhaps created by the shedding of vortices or the presence of wakes behind large particles (Yuan and Michaelides, 1992) which are not described by the fluid-particle interaction term in the present $k$ equation. This is the focus of our work in progress.

\section{Solid Velocity Fluctuation and Solids Distribution Profiles}

Figures Figures 11, 12a, and 13a compare the predictions for the fluctuating velocities of $200 \mu \mathrm{m}$ polystyrene spheres with previously unpublished experimental data by Tsuji (1993) at four 
different operating conditions. Solid velocity fluctuation data for particles larger than $200 \mu \mathrm{m}$ were not available as the number of instantaneous velocity measurements was not large enough to obtain accurate rms values. Figures $12 \mathrm{~b}$ and $13 \mathrm{~b}$ present the corresponding solids volume fraction profiles for which no experimental measurements were made. In all cases the agreement between the model predictions and the measurements for the solid velocity fluctuations is highly encouraging. The particle velocity fluctuations exceed those of the gas except in the near wall region and the magnitude of the random particle motion is modelled accurately using the kinetic theory analogy. It is clear that the particles do not follow the gas motions. For dilute phase flow, the solids distribution is essentially uniform. At the highest solids loadings investigated by Tsuji we predict a slight increase in the particle concentration at the tube axis which is reflected by a slight decrease in the particle fluctuation intensity there. This trend is also observed in the experimental data.

\section{Pressure Gradient Predictions}

In Figure 14 the relationship between the operating variables for the $500 \mu \mathrm{m}$ polystyrene spheres in the Tsuji system is investigated. Given a fixed solids mass flowrate, the pressure drop is accurately predicted over a range of radially-averaged mean gas velocities. The dashed line in Figure 14 indicates the pressure drop predictions from the simplified model which neglects gas phase turbulence but retains stresses in the particle phase. It is clear that turbulence in the gas phase plays an important role in the total pressure gradient.

\section{Sensitivity of the Model Predictions to the Coefficient of Restitution}

For the range of solids loadings considered in this study the model predictions are not extremely sensitive to the value of the coefficient of restitution as indicated in the previous studies of Sinclair and Jackson and Pita and Sundaresan. Figure 15 presents model predictions (some previously shown in Figure 4) for the $\mathbf{4 0 0} \mu \mathrm{m}$ spheres in the Lee and Durst system exploring a reasonable range of values for the coefficient of restitution $(0.85<e<0.97)$ for glass-glass 
contacts (Lun and Savage, 1986). A small change in the mean and fluctuating velocity profiles for both phases is noted. Similarly, minimal sensitivity in the predictions is observed (profiles not shown) when the coefficient of restitution for particle-wall collisions is varied over the same range. While the magnitude of the particle slip at the wall depends on the specularity factor, the model predictions are also not extremely sensitive to its value. When the specularity factor is increased by an order of magnitude, a variation of approximately $10 \%$ in the particle slip is observed.

\section{Sensitivity of the Model Predictions to the Constants Appearing in the k-E Equations}

In this work the model constants $c_{1}, c_{2}, c_{\mu}, \sigma_{k}$, and $\sigma_{\varepsilon}$ appearing in the $k-\varepsilon$ equations are the same values employed in the single phase turbulent flow model of Myong and Kasagi (1990). The use of these values is questionable for two-phase flows, even dilute-phase flow. The value for $\mathrm{c}_{3}$ follows Elghobashi and Abou-Arab (1983). . Thus the sensitivity of the model predictions to variations in the values for these constants is investigated. Deviations in the gas turbulent kinetic energy profiles are shown in Figures 16 and 17 when the model constants are altered over an interval of \pm 0.1 . The other flow variables are not shown as negligible changes in the results are obtained. Only the peak intensity of the $\mathrm{k}$ prediction is influenced by changes in $\mathrm{c}_{1}$ and $\mathrm{c}_{2}$. Overall the predictions do not reveal a large sensitivity to the values of the constants in the turbulence model.

\section{Conclusions}

In the present study we have expanded on the work of Sinclair and Jackson (1989) and Louge et al. (1991) to model the turbulent flow of a dilute gas-solid suspension. For the larger particles considered here, particle-particle collisions are significant and the fluctuating particle motion often exceeds that of the gas phase fluctuations. Because of the additional stresses in the solid phase due to these particle interactions, the observed centerline slip is larger than what would be given by a description of the two-phase flow which neglects particle-particle interactions. Comparisons between model predictions and experimental data for the mean and fluctuating solid 
velocity profiles indicate that a model based on the kinetic theory of granular materials accurately describes the particle flow in this situation. The model captures the progressive increase in slip velocity between the phases with increasing particle size, and the decrease in centerline slip with increasing solids loading for the same particle size. The model predictions also reproduce the flattening of the mean and fluctuating gas velocity profiles with increased solids loadings. Finally, for a fixed solids flux, the models predictions reflect the correct increase in pressure drop with increasing superficial gas velocity. The model failed, however, to quantitatively predict the degree of gas turbulence modulation due to the addition of particles. This could perhaps be remedied by including a mechanism for turbulence generation in the gas phase resulting from the shedding of vortices or the presence of wakes behind particles, but this remains to be demonstrated. It is shown that predictions from simplified models which neglect gas phase turbulence or particle phase stresses associated with particle-particle interactions do not match the observed flow behavior. For the range of solid loading ratios investigated in this study, the model does not manifest an extreme sensitivity to the coefficient of restitution for particle-particle or particle-wall collisions or the specularity factor. It should be emphasized that further experimentation employing non-intrusive flow measurement techniques are critical to understanding and developing predictive models for gas-solid flows.

\section{Acknowledgement}

The authors gratefully acknowledge funding support from the DOE University Coal Research Program Grant No. DE-FG22-92PC92540, the Dow Chemical Company, the ACS Petroleum Research Fund Grant No. 23854-G7, and the Pittsburgh Supercomputer Center. The authors would also like to thank Professor Tsuji for kindly providing the experimental measurements of the solid velocity fluctuations. 


\begin{tabular}{|c|c|}
\hline$\beta$ & drag coefficient \\
\hline $\boldsymbol{\varepsilon}$ & rate of dissipation of gas phase turbulent kinetic energy \\
\hline$\phi$ & specularity coefficient \\
\hline $\boldsymbol{\gamma}$ & rate of dissipation of pseudo-thermal energy per unit volume \\
\hline $\mathbf{P g}$ & gas density \\
\hline ps & solid density \\
\hline$\Phi$ & particle collisional stress tensor \\
\hline$\sigma_{\mathbf{k}}$ & turbulent Prandtl number for $k$ \\
\hline$\sigma_{\varepsilon}$ & turbulent Prandtl number for $\varepsilon$ \\
\hline I T & total stress tensor for the gas phase \\
\hline$v$ & solids volume fraction \\
\hline vo & solids volume fraction at closest packing \\
\hline$\mu$ & intrinsic gas viscosity \\
\hline$\mu_{t}$ & turbulent or eddy gas viscosity \\
\hline
\end{tabular}




\section{Notation}

$c_{\mu}, c_{1}, c_{2}, c_{3}$ constants in the $k-\varepsilon$ model

d particle diameter

e coefficient of restitution for particle-particle collisions

Cw coefficient of restitution for particle-wall collisions

$f_{\mu}, f_{1}, f_{2}$, functions in $k-\varepsilon$ model

k gas phase turbulent kinetic energy

m solids loading (solid-to-gas mass flow ratio)

p pressure

qPT flux vector of pseudo-thermal energy for the particle phase

r radial coordinate

R pipe radius

Re Reynolds number based on the pipe diameter

Red Reynolds number based on the particle diameter, $\operatorname{Re}_{d}=(1-v) d \mid v_{z}-u_{z}$

RT turbulent Reynolds number, $\mathrm{RT}=\rho_{\mathrm{g}} \mathrm{k}^{2} /(\mu \varepsilon)$

T particle temperature

$\mathbf{U}_{\boldsymbol{\tau}} \quad$ friction velocity

u solid velocity

u' fluctuating solid velocity component

gas velocity

v' fluctuating gas velocity component

$\mathrm{Va}$ centerline gas velocity.

$y$ normal distance from the wall, $y=R-r$

$y^{+} \quad$ dimensionless distance from the wall, $y^{+}=\rho_{g} y U_{T} / \mu$

$\mathbf{z}$

axial coordinate 


\section{Literature Cited}

Anderson, T. and R. Jackson, "Fluid mechanical description of fluidized beds: Comparison with theory and experiment", I\&EC Fundamentals, 6, 527 (1967).

Chen, C. and P. Wood, "Turbulence closure model for dilute gas-particle flows", Can. J. of Chem. Engr., 63, 349 (1985).

Dasgupta, S., R. Jackson, and S. Sundaresan, "Turbulent gas-particle flow in vertical risers", AIChE J., 40, 215 (1994).

Ding, J. and D. Gidaspow, "A bubbling fluidization model using kinetic theory analogy of granular flow", AIChE J., 36, 523 (1990).

Elghobashi, S. and T. Abou-Arab, "A two-equation turbulence model for two-phase flows", Phys. Fluids, 26, 931 (1983).

Hrenya, C., E. Bolio, D. Chakrabarti, and J. Sinclair, "Comparative study of low Reynolds k- $\varepsilon$ models in predicting turbulent pipe flow", submitted for publication in Chem. Eng. Sci. (1993).

Hui, K., P. Haff, J. Ungar, and R. Jackson, "Boundary conditions for high shear grain flows", J. Fluid Mech., 145, 223 (1984).

Johnson, P. and R. Jackson, "Frictional-collisional constitutive relations for granular materials, with application to plane shearing", J. Fluid Mech., 176, 67 (1987). 
Koch, D., "Kinetic theory for a monodisperse gas-solid suspension", Phys. Fluids A, 2, 1711 ( 90).

Lee, S. and F. Durst, "On the motion of particles in turbulent duct flows", Int. J. Multiphase Flow, 8, 125 (1982).

Louge, M., E. Mastorakos, and J. Jenkins, "The role of particle collisions in pneumatic transport", J. Fluid Mech., 231, 345 (1991).

Lun, C., S. Savage, D. Jeffrey, and N. Chepumiy, "Kinetic theories for granular flow; Inelastic particles in couette flow and slightly inelastic particles in a general flow field", J. Fluid Mech., 140,223 (1984).

Lun, C. and S. Savage, "The effects of an impact velocity dependent coefficient of restitution on stresses developed by sheared granular materials", Acta Mechanica, 63, 15 (1986).

Maeda, M., K. Hishida, and T. Furutani, "Optical measurements of local gas and particle velocity in an upward flowing dilute gas-solids suspension", Polyphase Flow and Transport Technology, Century 2-ETC, 211 (1980).

Martinuzzi, R. and A. Pollard, "Comparative study of turbulence models in predicting turbulent pipe flow", AIAA J., 27, 29 (1989).

Myong, H. and N. Kasagi, "A new approach to the improvement of k-e turbulence model for wallbounded shear flows", JSME Int. J. Series II, 33, 63 (1990). 
Ocone, R., S. Sundaresan, and R. Jackson, "Gas-paricle flow in a duct of arbitrary inclination with particle-particle interactions", AIChE J., 39, 1261 (1993).

Patankar, S. and D. Spalding, Heat and Mass Transfer in Boundary Layers, MorganGrampian Presi, London (1967).

Pita, J. and S. Sundaresan, "Gas-solid flow in vertical tubes", AIChE J., 37, 1009 (1991).

Pita, J. and S. Sundaresan, "Developing flow of a gas-particle mixture in a vertical riser", AIChE J., 39, 541 (1993).

Pourahmadi, F. and J. Humphrey, "Modelling solid-fluid turbulent flows with application to predicting erosive wear", PhysicoChemical Hydrodynamics, 4, 191 (1983).

Rizk, M. and S. Elghobashi, "A two-equation turbulence model for dispersed dilute confined twophase flows", Int. J. Multiphase Flow, 15, 119 (1989).

Schildknecht, M., J. Miller, and G. Meier, "The influence of suction on the structure of turbulence in fully-developed pipe flow", J. Fluid Mech., 90, 67 (1979).

Shaffer, F., H. Massah, J. Sinclair, and M. Shahnam, "Measurement of time-averaged particlewall collision properties using particle tracking velocimetry", Proceedings of the First Intemational Particle Technology Forum, Denver, Colorado (1994).

Sinclair, J. and R. Jackson, "Gas-particle flow in a vertical pipe with particle-particle interactions", AIChE J., 35, 1473 (1989). 
Tsuji, Y., Y. Morikawa, and H. Shiomi, "LDV measurements of an air-solid two-phase flow in a vertical pipe", J. Fluid Mech., 139, 417 (1984).

Tsuji, Y., Private communication (1993).

Yuan, Z. and E. Michaelides, "Turbulence modulation in particulate flows-A theoretical approach", Int. J. Multiphase Flow, 18, 779 (1992). 
Table 1

Summary of Model Constants and Functions Appearing in the k- $\varepsilon$. Equations

$$
\begin{aligned}
& c_{1}=1.4 \\
& c_{2}=1.8 \\
& c_{3}=1.2 \\
& c_{\mu}=0.09 \\
& \sigma_{k}=1.4 \\
& \sigma_{\varepsilon}=1.3 \\
& f_{1}=1.0 \\
& f_{2}=\left\{1-\frac{2}{9} \exp \left[-\left(\frac{R_{T}}{6}\right)\right]\right\}\left[1-\exp \left(-\frac{y^{+}}{5}\right)\right]^{2} \\
& f_{\mu}=\left\{1-\exp \left[-\frac{y^{+}}{70}\right]\right\}_{j}\left[1+3.45 / \sqrt{R_{T}}\right]
\end{aligned}
$$


Table 2

Closures for $\mathrm{g}, \mathrm{g}_{\mathrm{pT}}$, and $\gamma$

$$
\begin{gathered}
\sigma_{\mathrm{rz}}=-\mu_{s}\left(\omega g_{1}+g_{2}\right) \frac{\partial u_{2}}{\partial r} \quad \sigma_{\mathrm{rr}}=\sigma_{\theta \theta}=\rho_{s}\left(\omega v+4 \eta v^{2} g_{0}\right) T \\
g_{1}=\frac{1}{\eta(2-\eta) g_{0}}\left[1+\frac{8}{5} \eta v g_{0}(3 \eta-2)\right] \\
=\frac{1}{1+\lambda_{\text {mfp }} / R}=\frac{8 v}{5(2-\eta)}\left[1+\frac{8}{5} \eta v g_{0}(3 \eta-2)\right]+\frac{768 v^{2} g_{0} \eta}{25 \pi} \\
g_{s}=\frac{5 \sqrt{\pi} d \rho_{s} \sqrt{T}}{96} g_{0}=\frac{v_{l}^{1 / 3}}{v_{0}^{1 / 3}-v^{1 / 3}} \eta=\frac{1+e}{2}
\end{gathered}
$$

$$
\begin{gathered}
q_{\mathrm{PT}}=-\lambda\left(\omega \mathrm{g}_{3}+\mathrm{g}_{4}\right) \frac{\partial T}{\partial \mathrm{r}} \quad \lambda=\frac{25 \sqrt{\pi} \mathrm{d} \rho_{8} \sqrt{T}}{128} . \\
\mathrm{g}_{3}=\frac{8}{\eta(41-33 \eta) g_{0}}\left[1+\frac{12}{5} \eta^{2} \vee g_{0}(4 \eta-3)\right] \\
g_{4}=\frac{96 v}{5(41-33 \eta)}\left[1+\frac{12}{5} \eta^{2} \vee g_{0}(4 \eta-3)+\frac{16}{15 \pi} \eta v g_{0}(41-33 \eta)\right]
\end{gathered}
$$$$
\gamma=\frac{48}{\sqrt{\pi}} \eta(1-\eta) g_{0} v^{2} \frac{\rho_{8}}{d} T^{3 / 2}
$$ 


\section{List of Figures}

1. Radial variation of (a) gas turbulent kinetic energy and (b) r.m.s. axial gas velocity fluctuations. The circles represent the data of Schildknecht et al. (1979).

2. Radial variation of mean gas and solid velocity. The circles represent the data of Lee and Durst (1982) for $100 \mu \mathrm{m}$ glass spheres. The dashed line represents predicted solid velocities from an analysis which neglects shear stress in the particle phase.

3. Radial variation of mean gas and solid velocity. The circles represent the data of Lee and Durst (1982) for $200 \mu \mathrm{m}$ glass spheres. The dashed line represents predicted solid velocities from an analysis which neglects shear stress in the particle phase.

4. Radial variation of mean gas and solids velocity. The circles represent the data of Lee and Durst (1982) for $400 \mu \mathrm{m}$ glass spheres. The dashed line represents predicted solid velocities from an analysis which neglects shear stress in the particle phase.

5. Radial variation of mean gas and solids velocity. The circles represent the data of Maeda et al. (1980) for $45 \mu \mathrm{m}$ glass spheres. The dashed lines represent predicted velocities from an analysis which neglects shear stress in the particle phase.

6. Radial variation of (a) mean gas velocity and (b) mean solids velocity. The circles represent the data of Maeda et al. (1980) for $136 \mu \mathrm{m}$ glass spheres. The dashed lines represent predictions from an analysis which neglects shear stress in the particle phase. The lines with solid squares represent predictions from an analysis which neglects shear stress in the particle phase and gas phase turbulence. 
7. Radial variation of (a) mean gas velocity and (b) mean solids velocity. The circles represent the data of Tsuji et al. (1984) for $200 \mu \mathrm{m}$ polystyrene spheres at $\mathrm{m}=1.0$. The dashed lines represent predictions from an analysis which neglects gas phase turbulence.

8. Radial variation of (a) mean gas velocity and (b) mean solids velocity. The circles represent the data of Tsuji et al. (1984) for $500 \mu \mathrm{m}$ polystyrene spheres at $\mathrm{m}=1.1$. The dashed lines represent predictions from an analysis which neglects gas phase turbulence.

9. Radial variation of (a) mean gas velocity and (b) mean solids velocity. The circles represent the data of Tsuji et al. (1984) for $200 \mu \mathrm{m}$ polystyrene spheres at $\mathrm{m}=4.2$. The dashed lines represent predictions from an analysis which neglects the kinetic contribution to the particle phase stress.

10. Radial variation of r.m.s axial gas velocity fluctuations at solids loadings of (a) $m=0.9$ and (b) $m=3.2$. The circles represent the data of Tsuji et al. (1984) for $200 \mu \mathrm{m}$ polystyrene spheres. The dashed lines represent the clear gas predictions at the same Reynolds number.

11. Radial variation of r.m.s solid velocity fluctuations at solids loadings of (a) $m=1.3$ and (b) $m=3.2$. The circles represent the data of Tsuji et al. (1984) for $200 \mu \mathrm{m}$ polystyrene spheres.

12. Radial variation of (a) r.m.s solid velocity fluctuations and (b) solids volume fraction at $m=1.0$. The circles represent the data of Tsuji et al. (1984) for $200 \mu \mathrm{m}$ polystyrene spheres.

13. Radial variation of (a) r.m.s solid velocity fluctuations and (b) solids volume fraction at $m=4.2$. The circles represent the data of Tsuji et al. (1984) for $200 \mu \mathrm{m}$ polystyrene spheres. 
14. Variation of pressure gradient with gas velocity at a fixed solids mass flowrate $(0.03 \mathrm{~kg} / \mathrm{s})$. The circles represent the data of Tsuji et al. (1984) for $500 \mu \mathrm{m}$ polystyrene spheres. The dashed line represents predictions from an analysis which neglects gas phase turbulence.

15. Sensitivity analysis. Radial variation of the (a) mean velocities and (b) fluctuating velocities for three different values of the coefficient of restitution for particle-particle collisions. Operating conditions are the same as in Figure 4.

16. Sensitivity analysis. Radial variation of the r.m.s. axial gas velocity fluctuations for three different values of the model constants $(a) c_{1}(b) c_{2}(c) c_{3}$. Operating conditions are the same as in Figure 10b.

17. Sensitivity analysis. Radial variation of the r.m.s. axial gas velocity fluctuations for three different values of the model constants $(\dot{a}) c_{\mu}(b) \sigma_{k}(c) \sigma_{\varepsilon}$. Operating conditions are the same as in Figure 10b. 


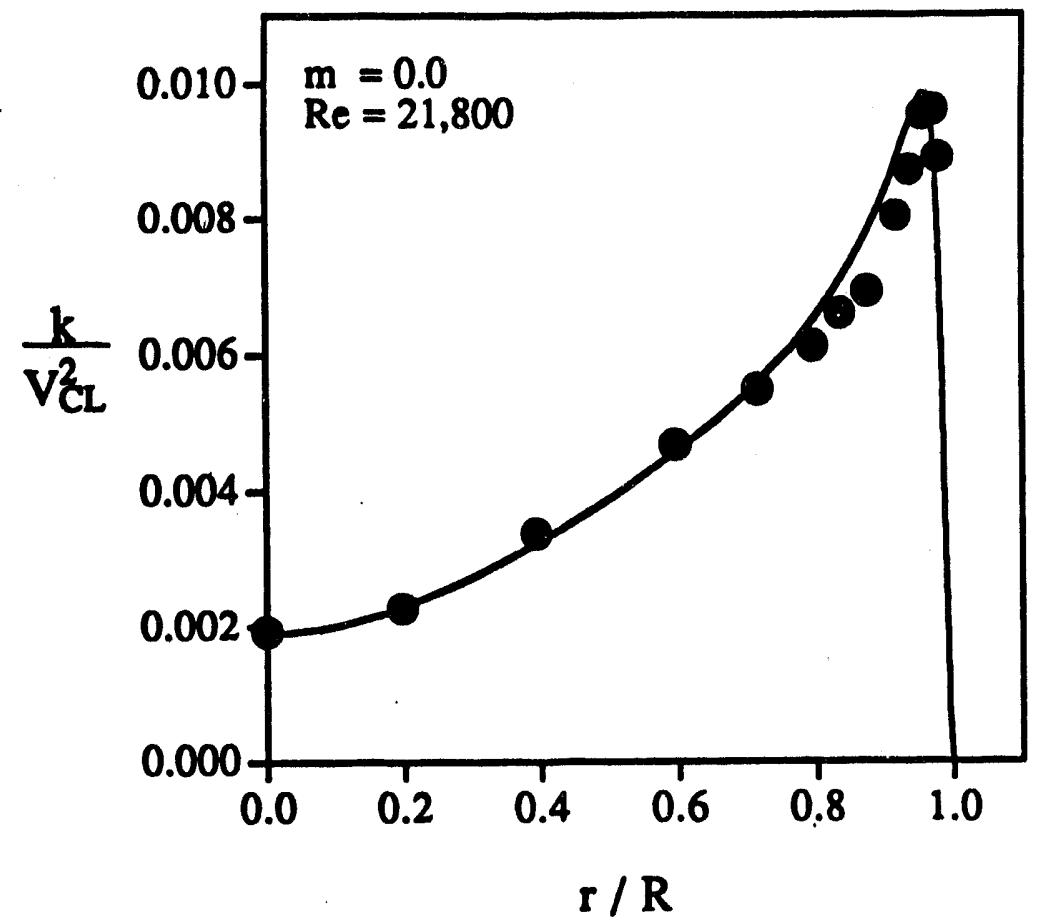

(a)

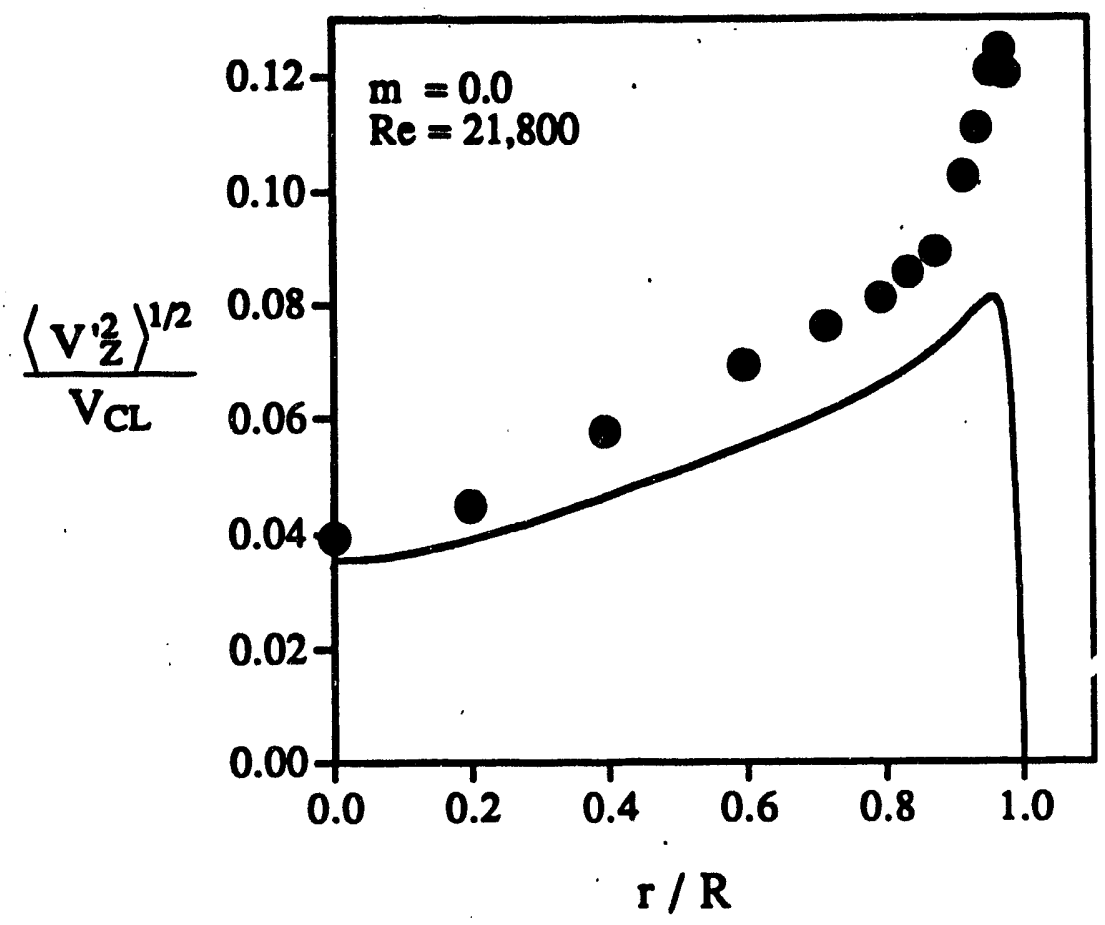

(b) 


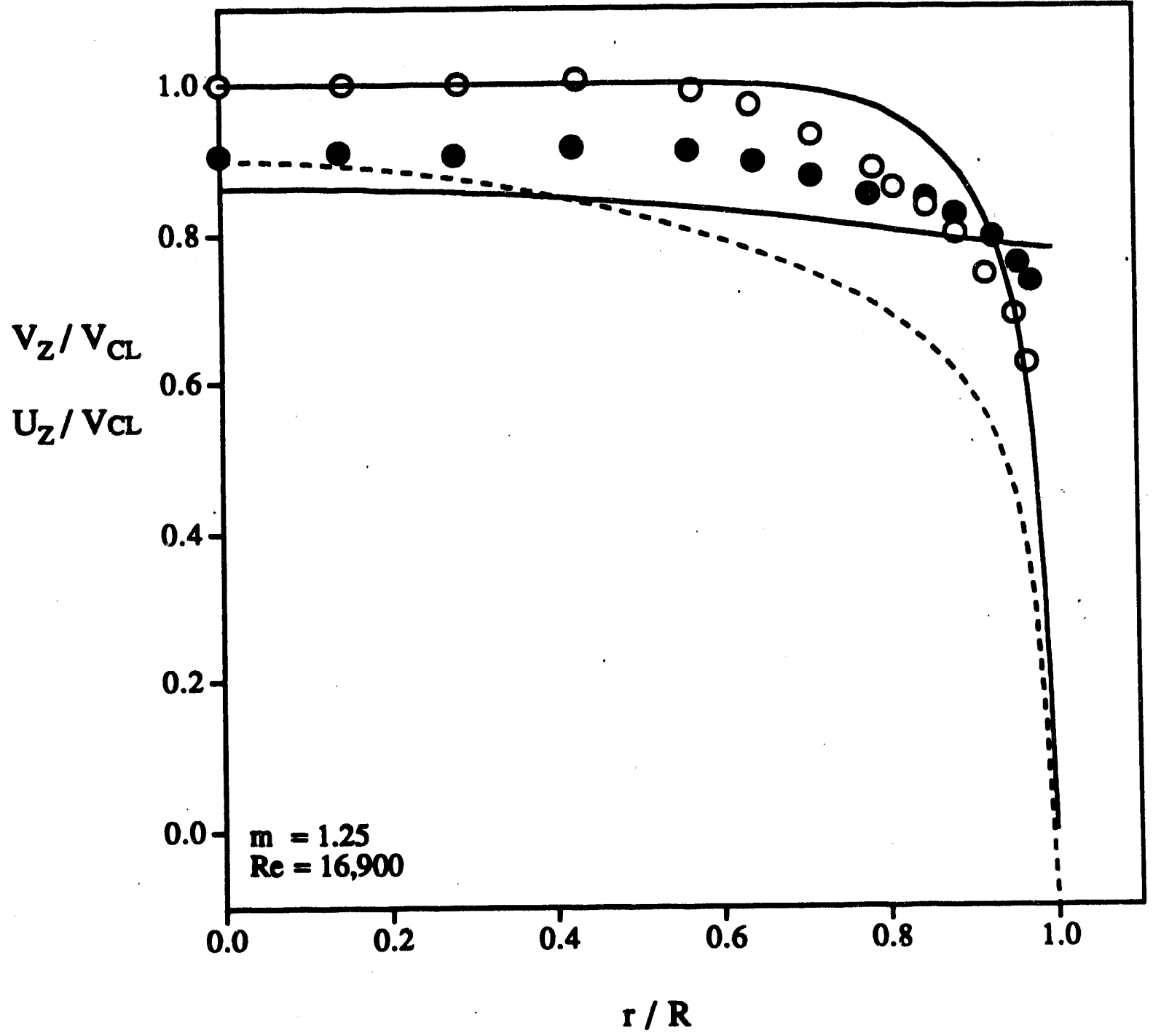

FIGURE 2 


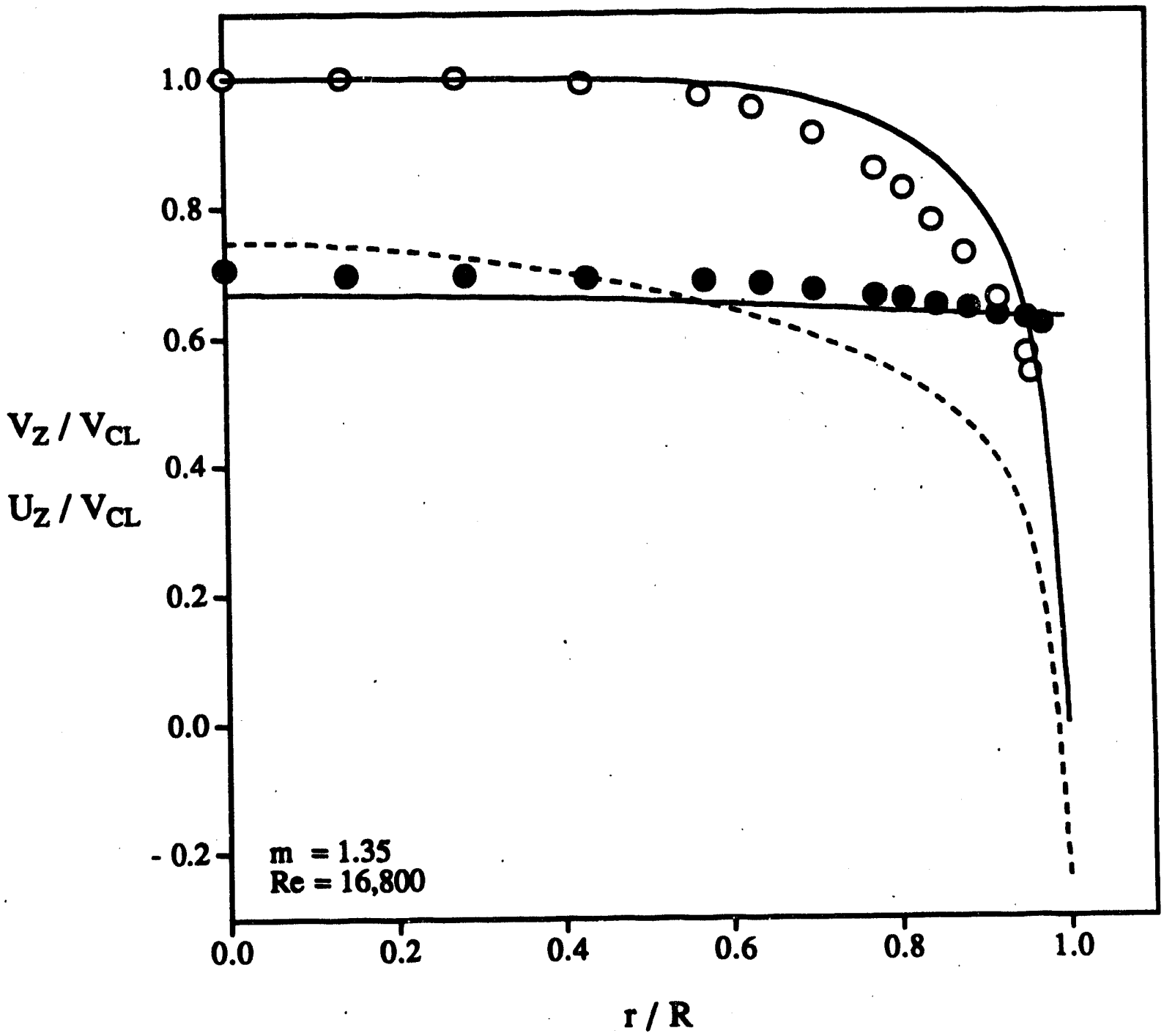

FIGURE 3 


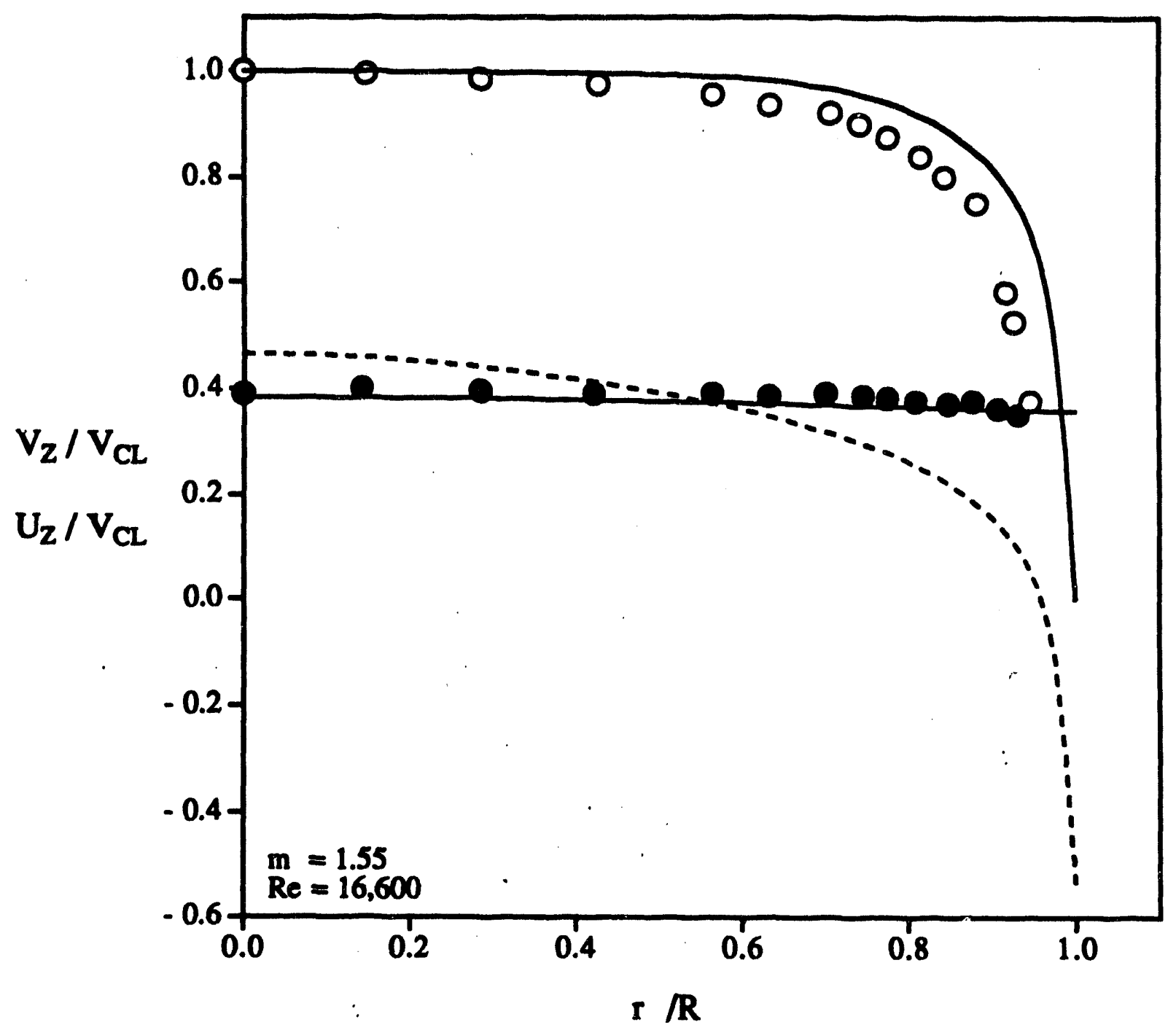

FIGURE 4 


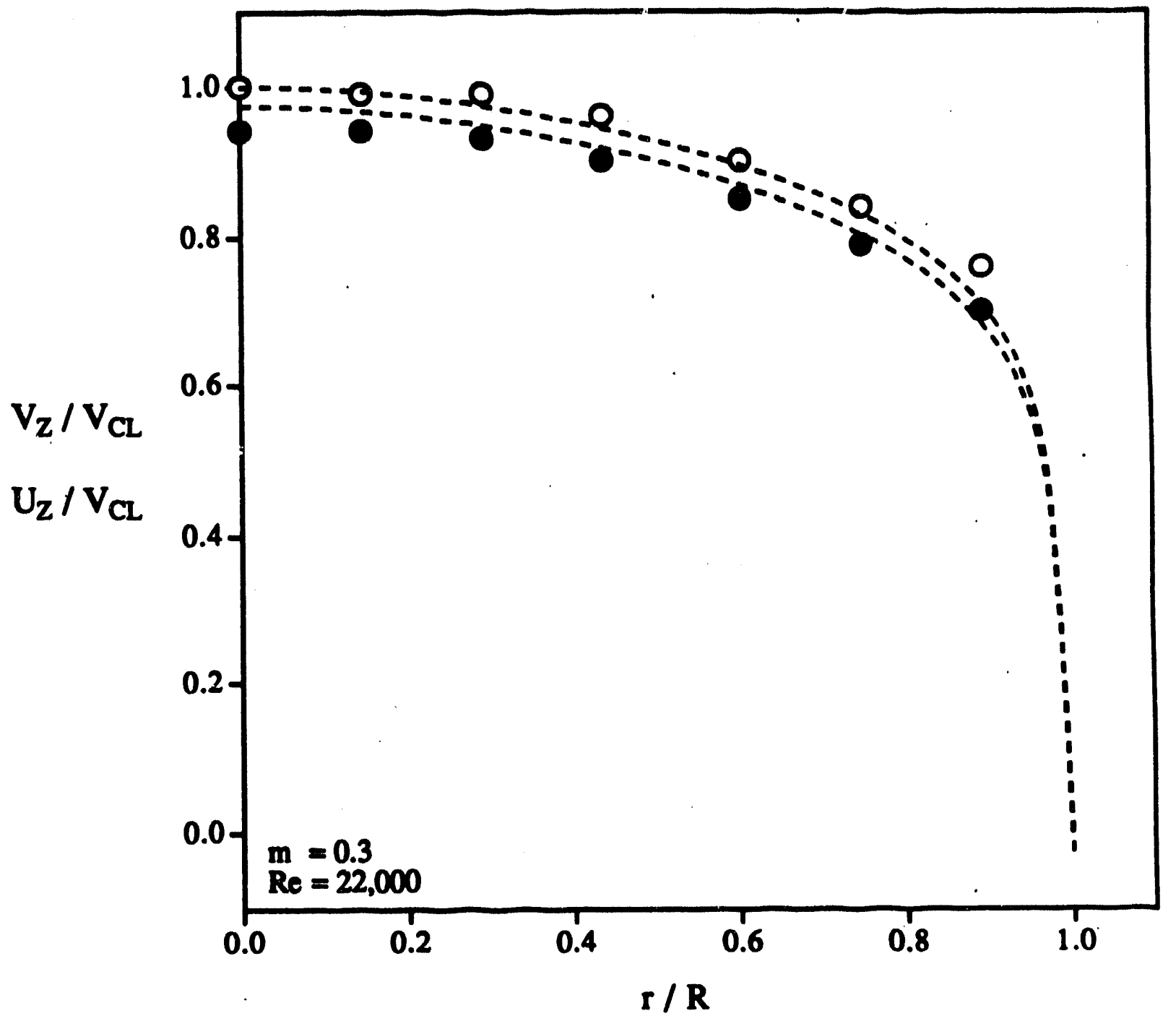

FIGURE 5 


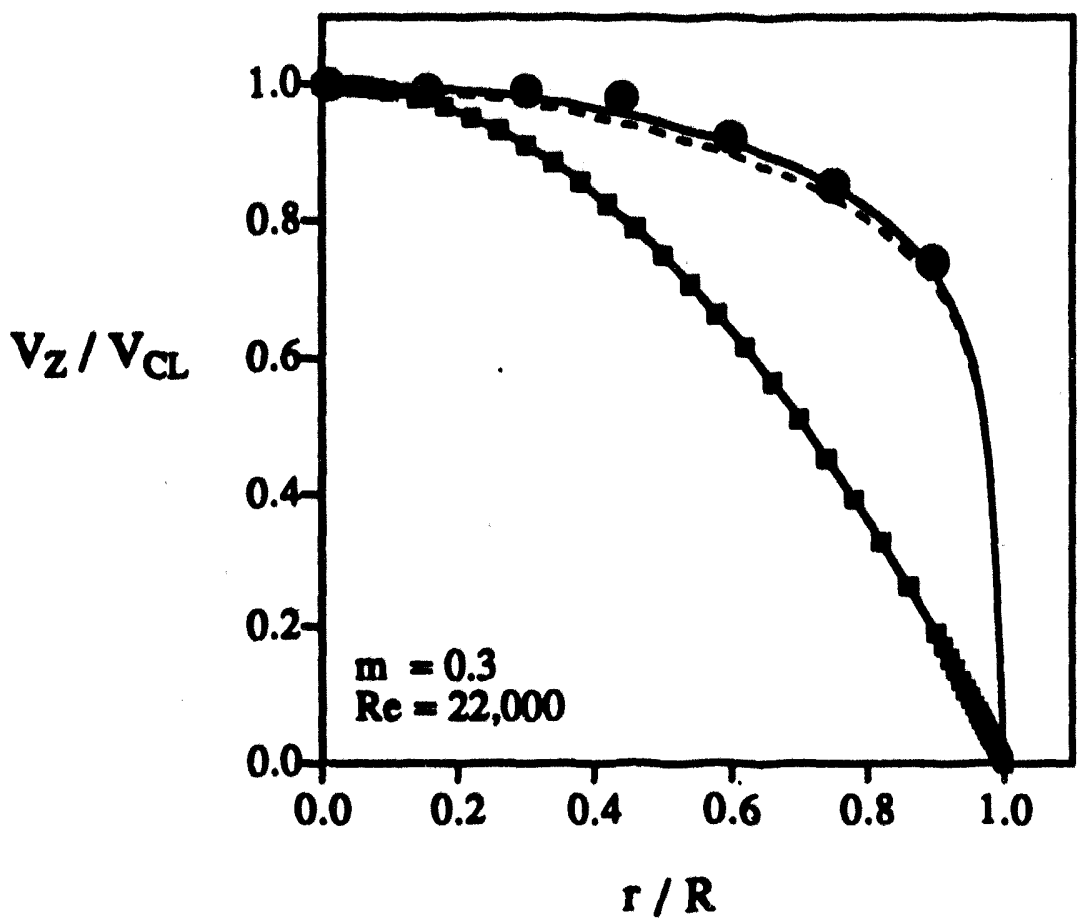

(a)

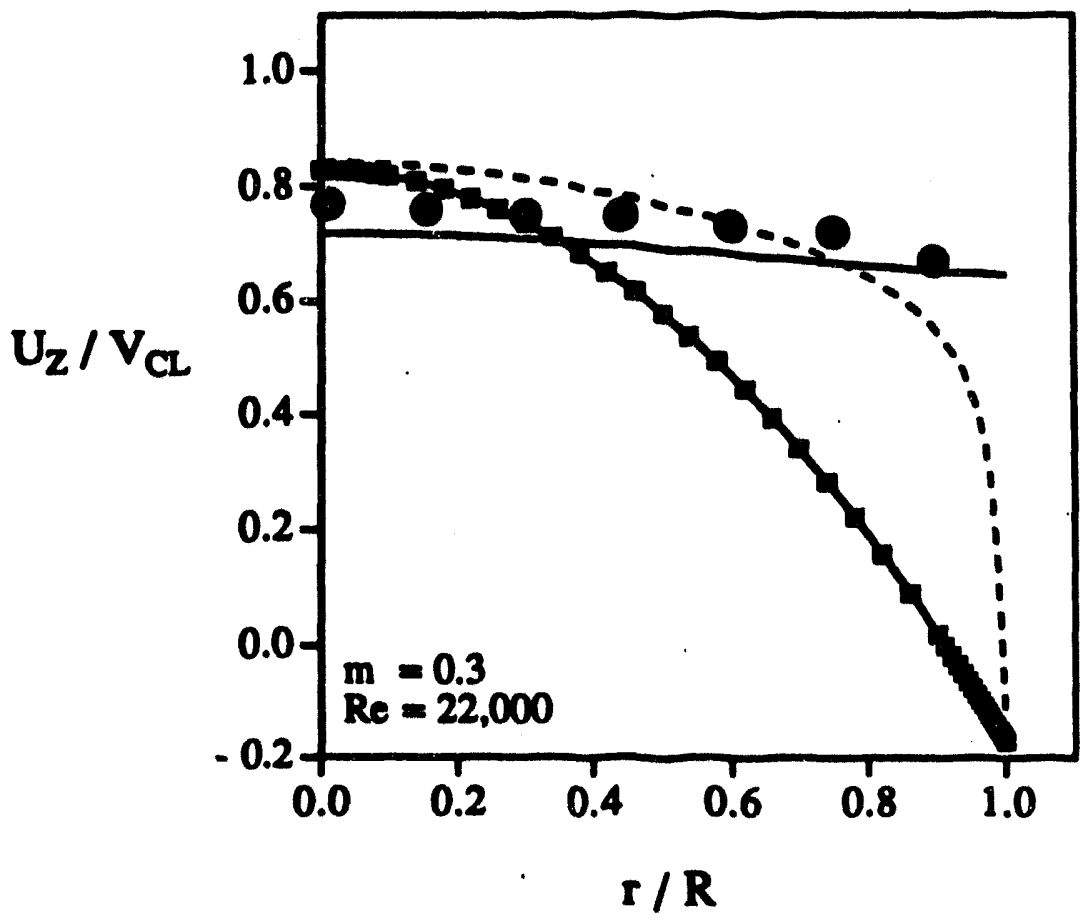

(b)

FIGURE 6 


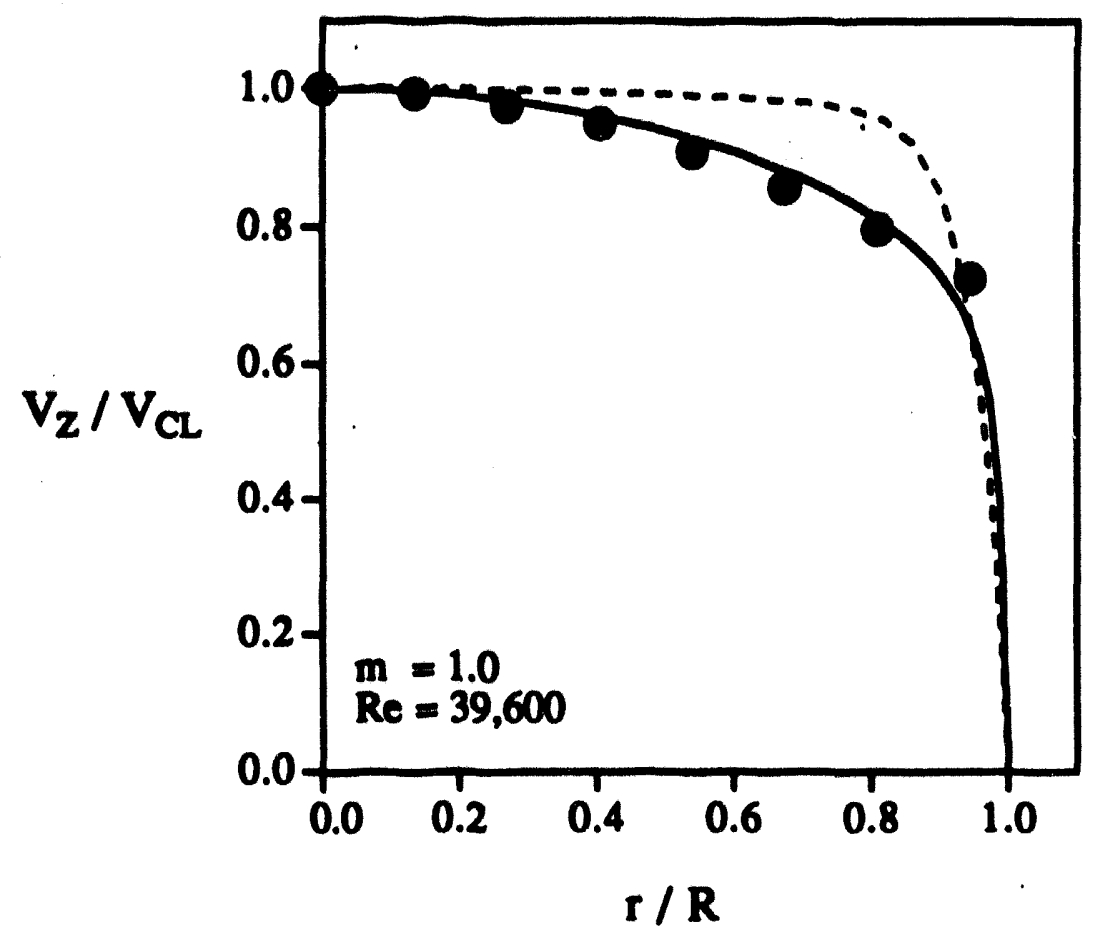

(a)

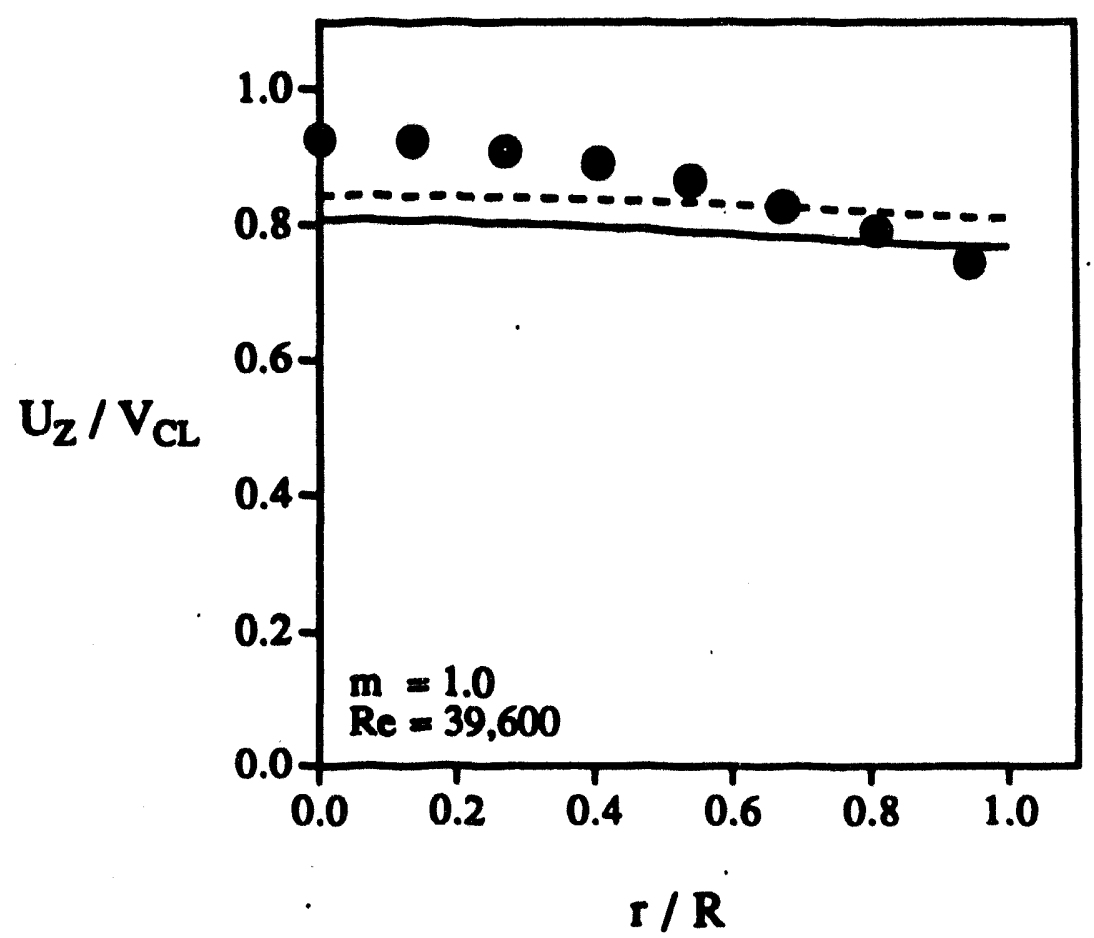

(b)

FIGURE 7 


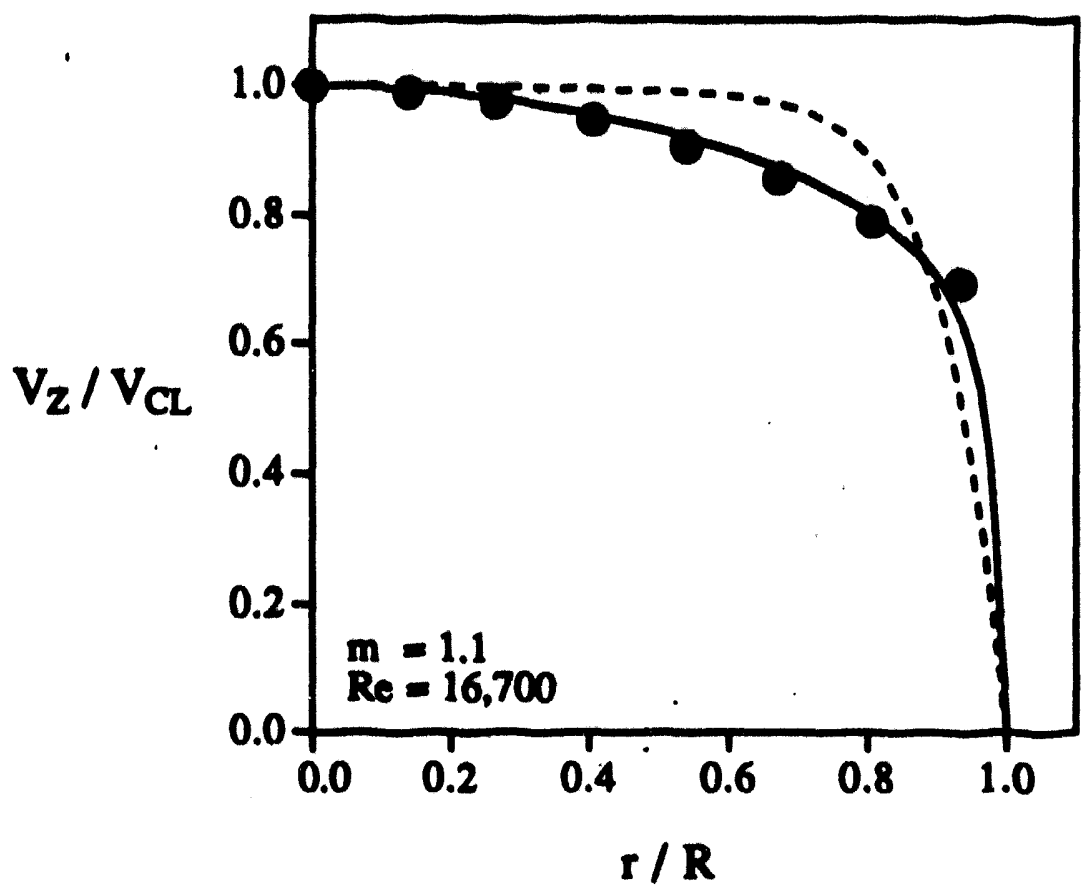

(a)

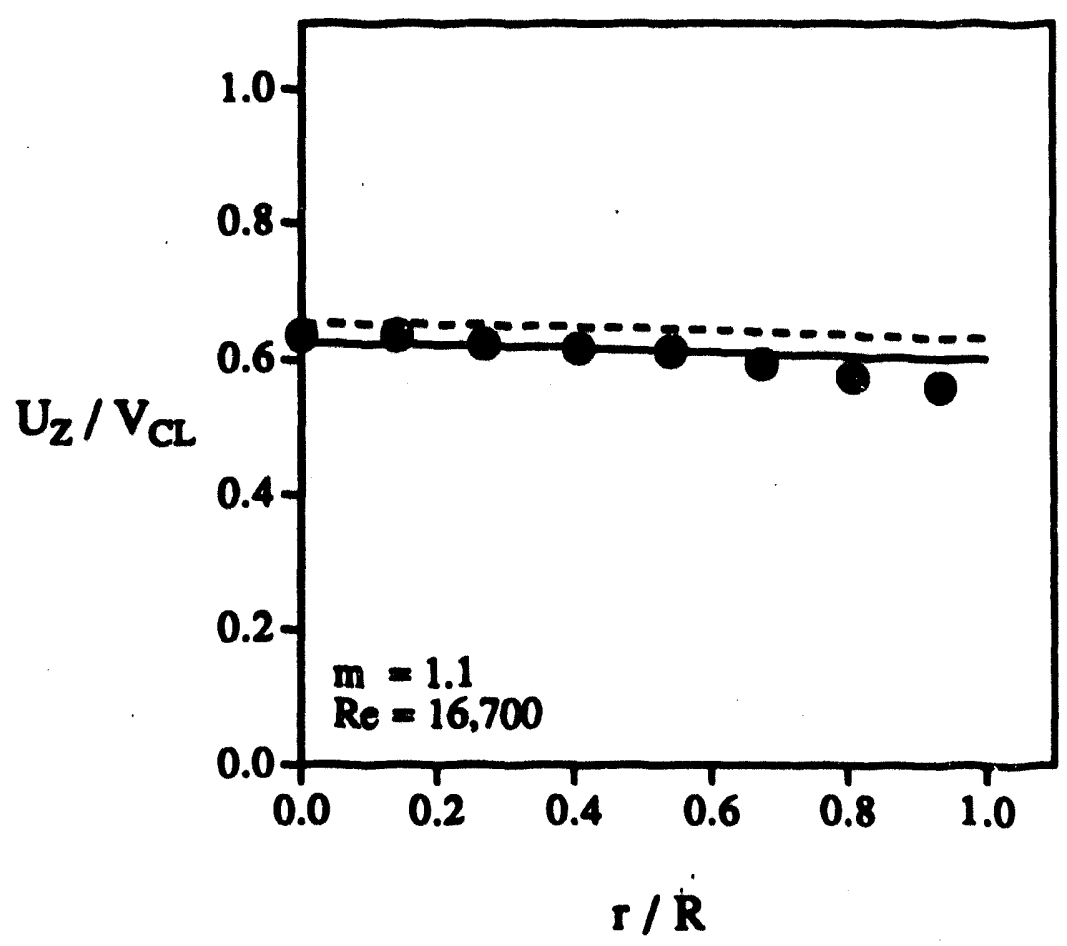

(b) 


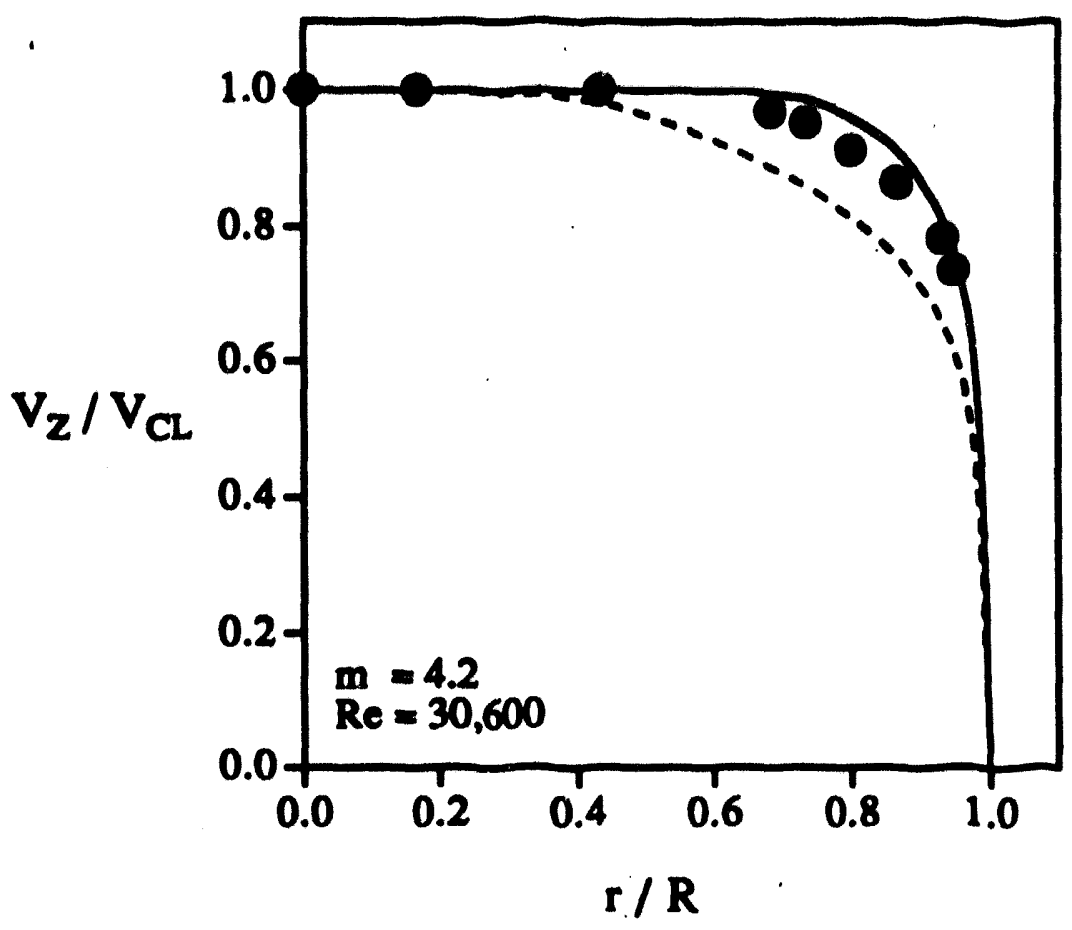

(a)

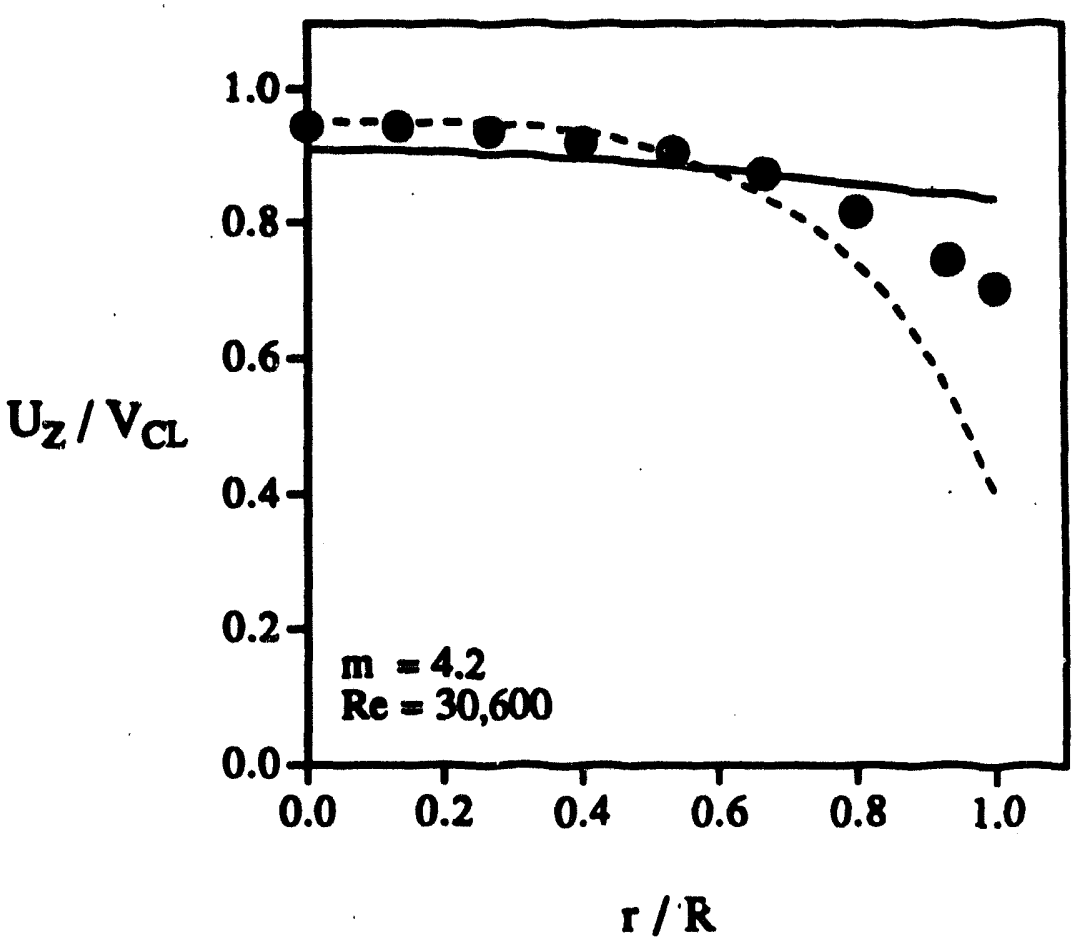

(b) 


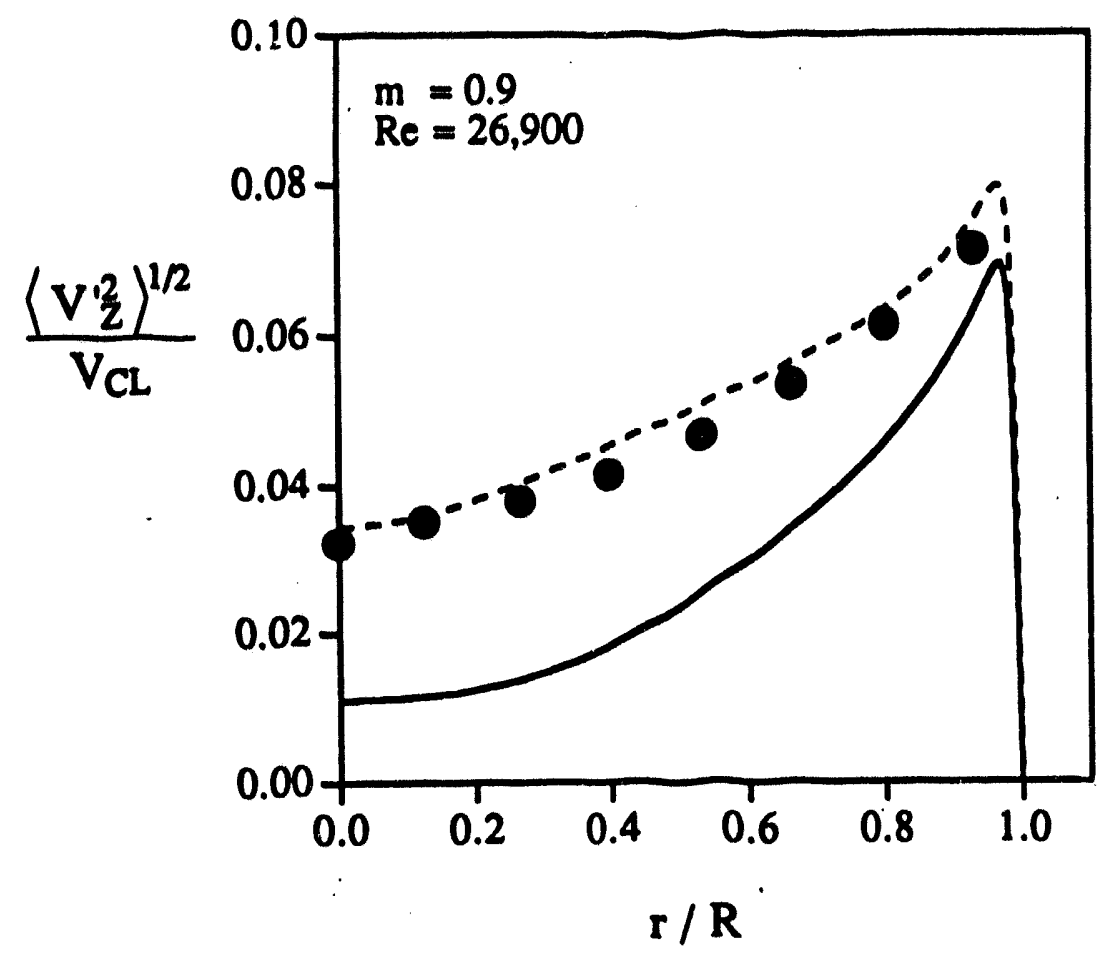

(a)

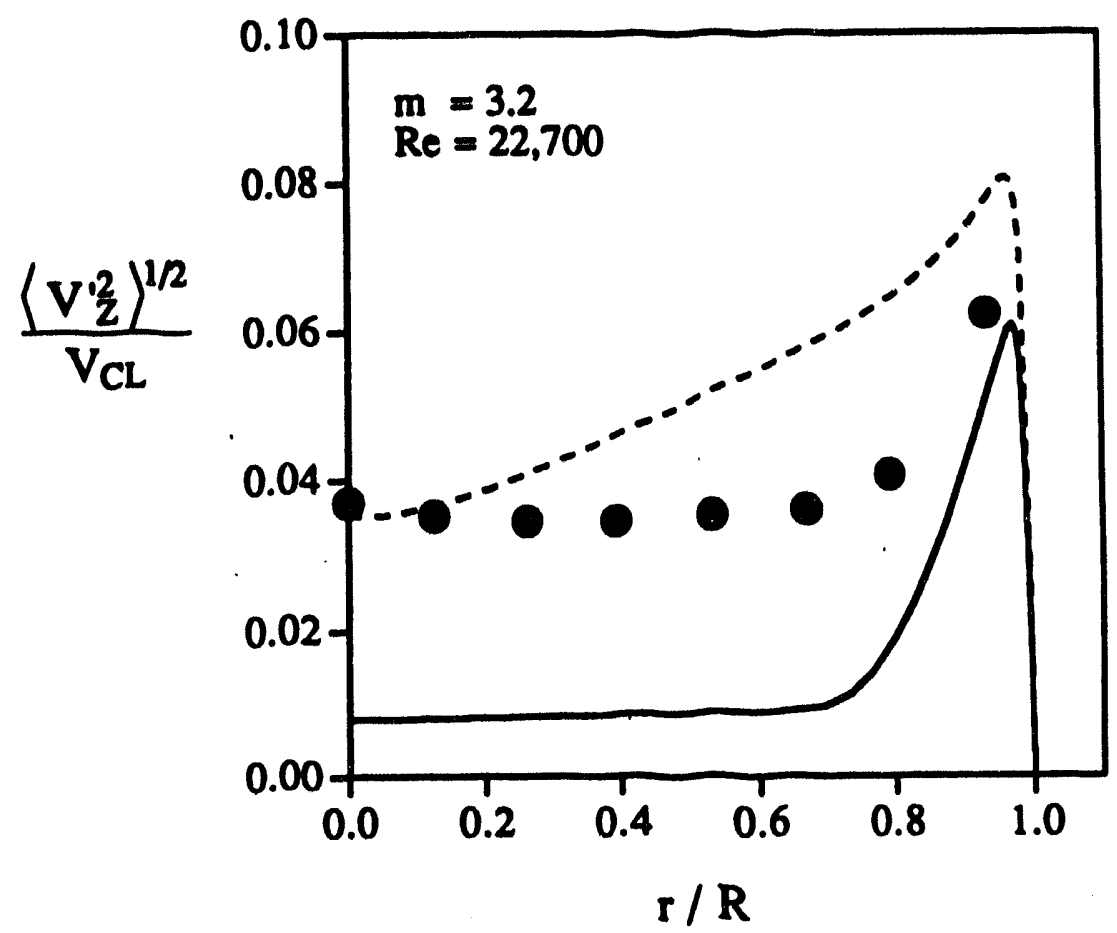

(b) 


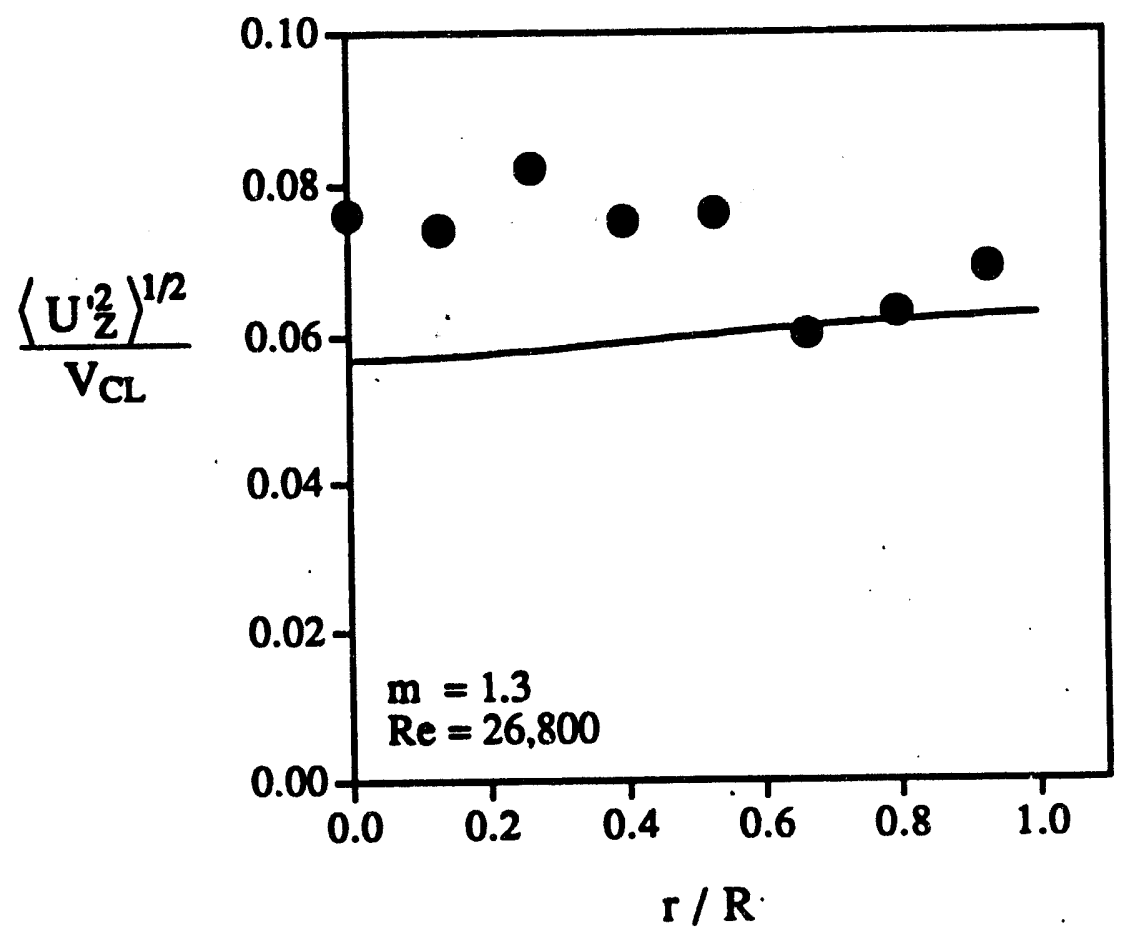

(a)

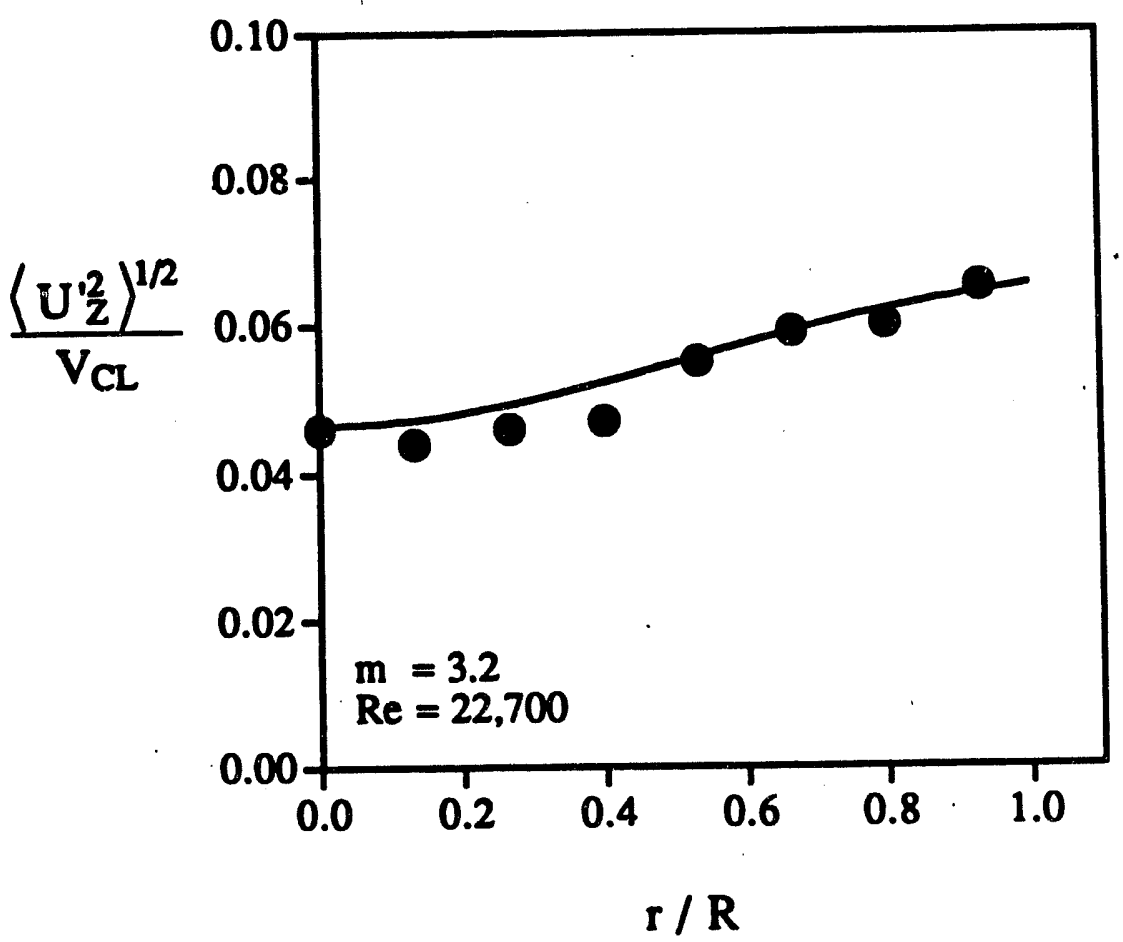

(b) 


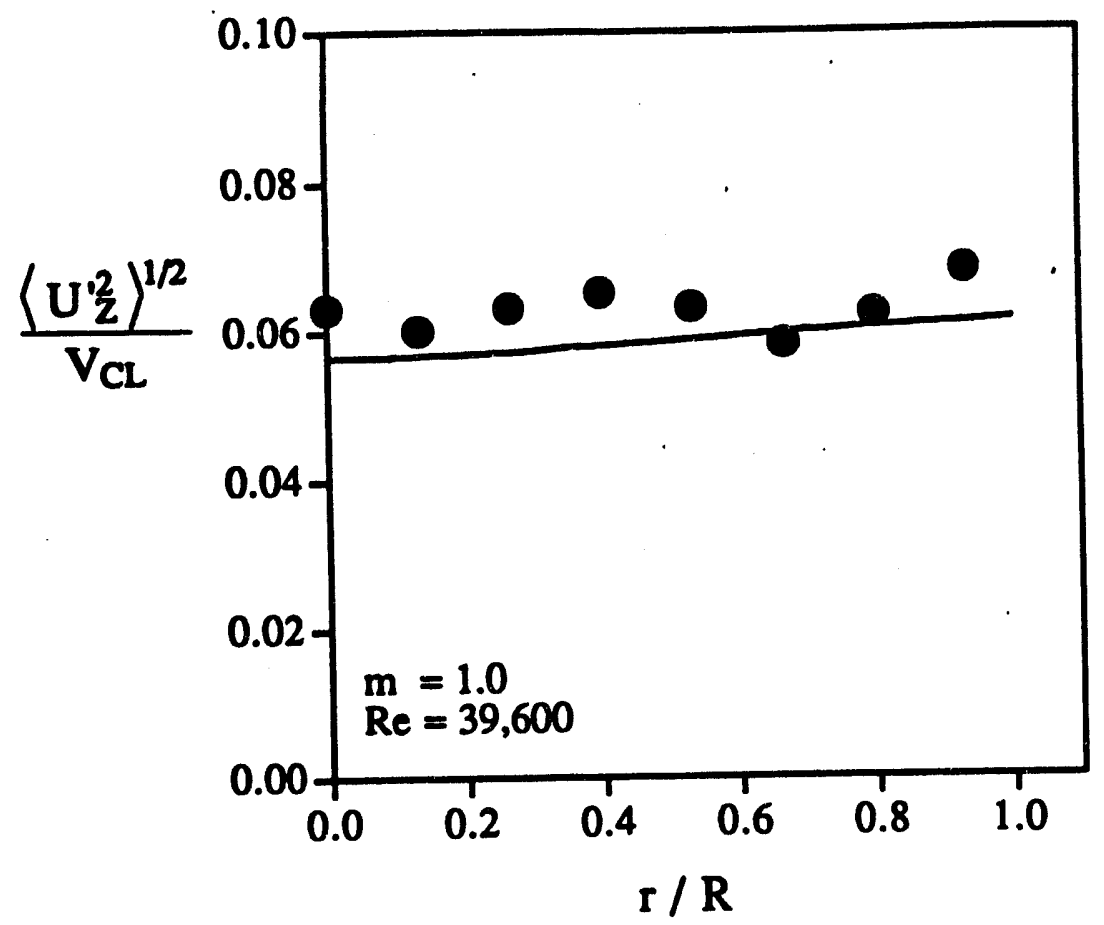

(a)

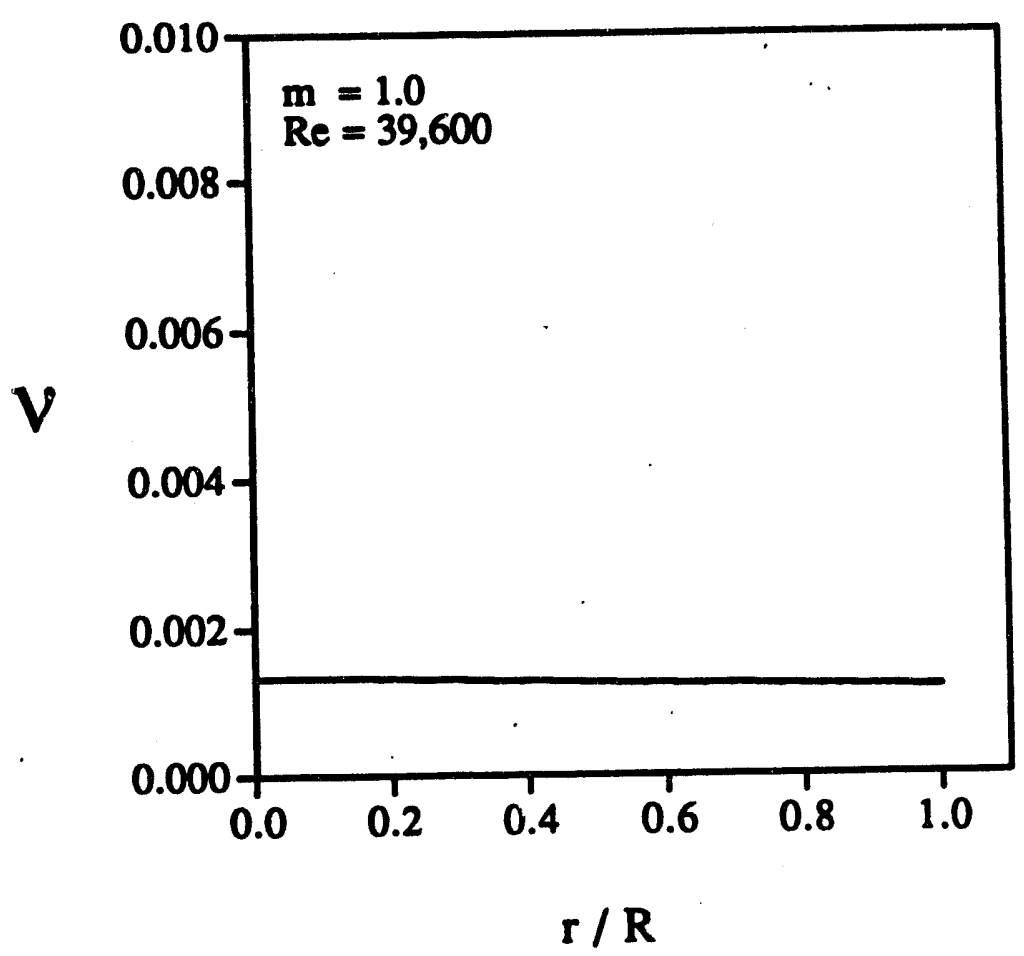

(b) 


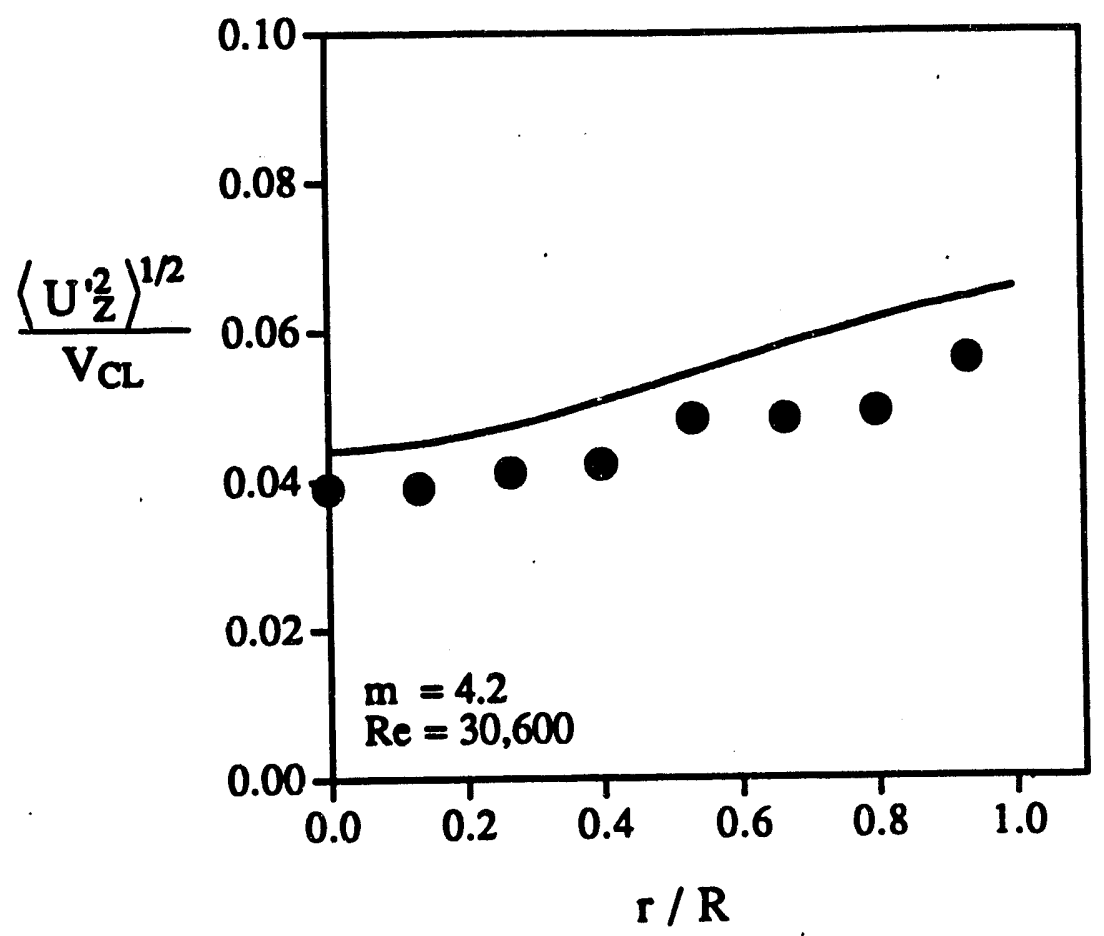

(a)

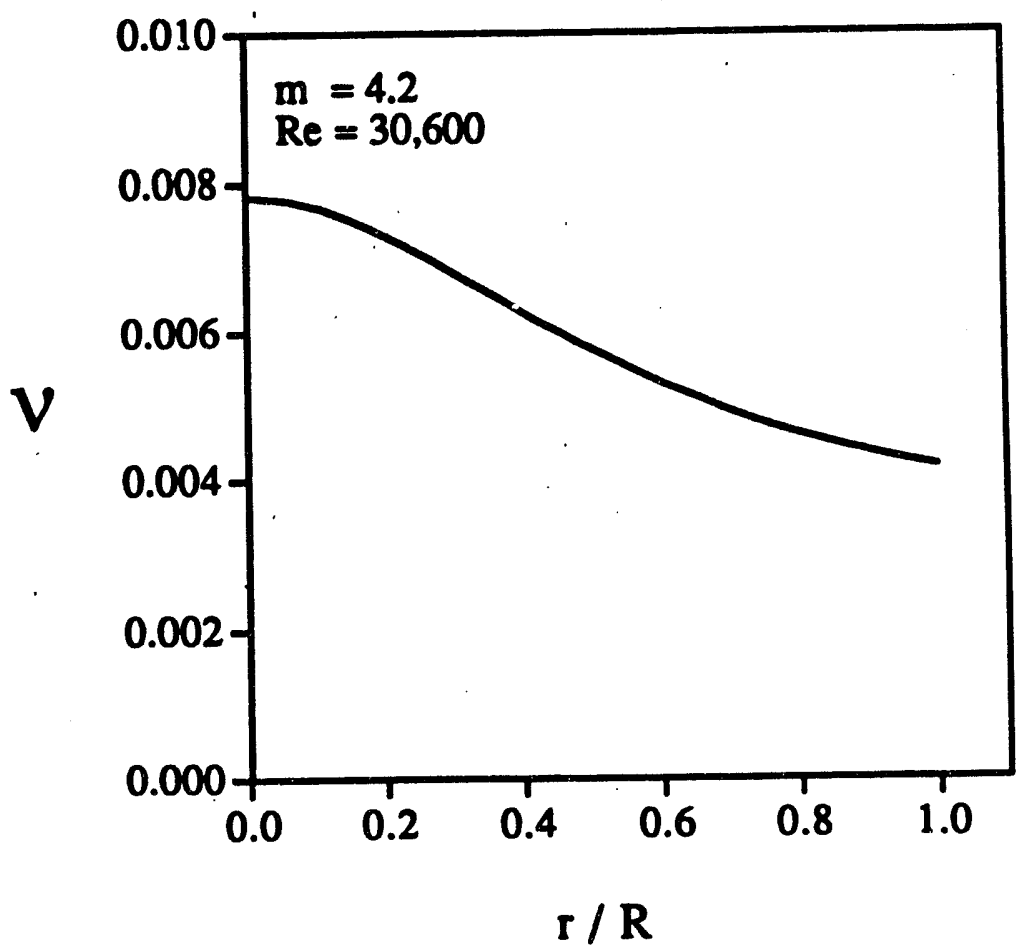

(b) 


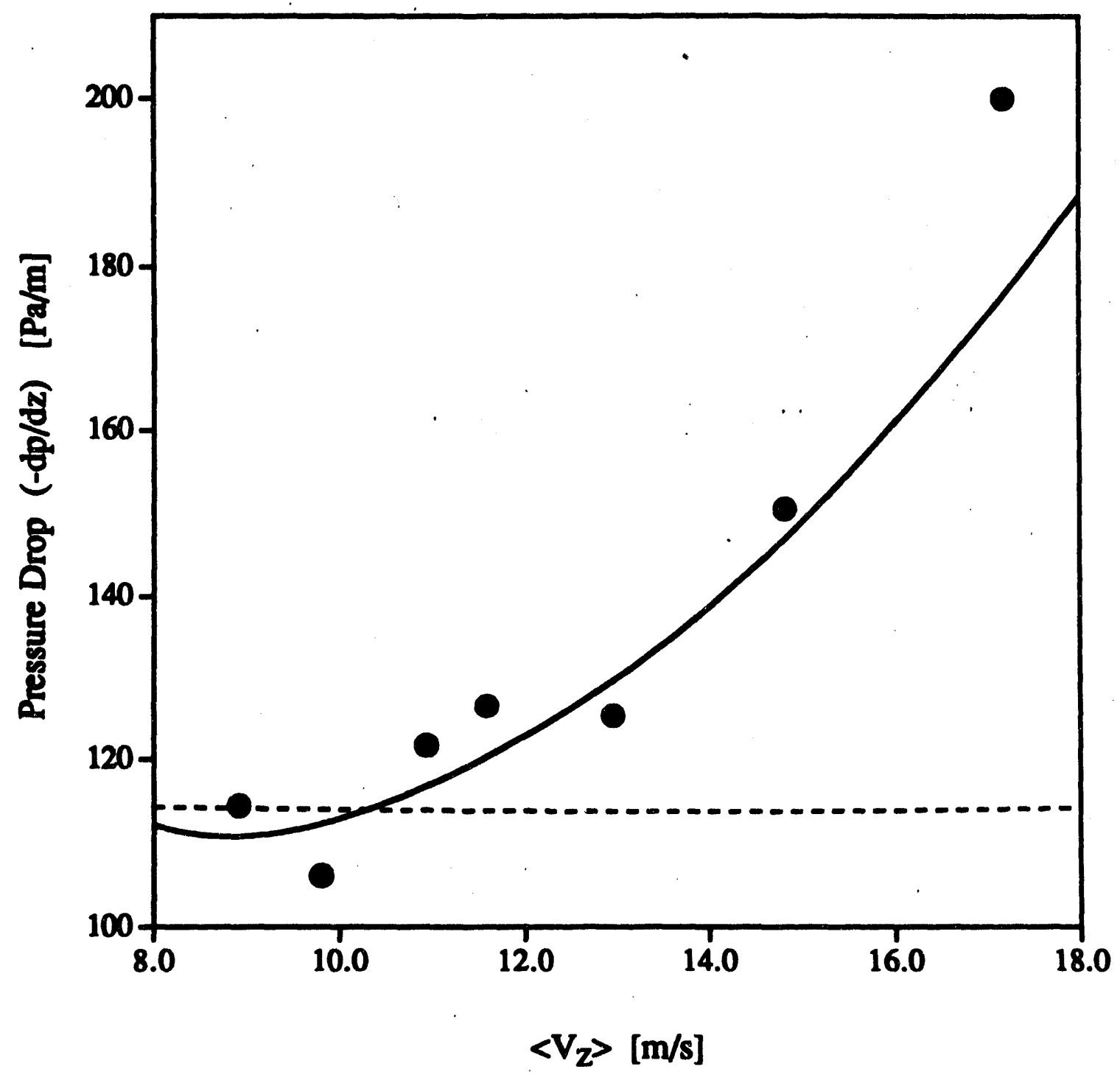

FIGURE 14 


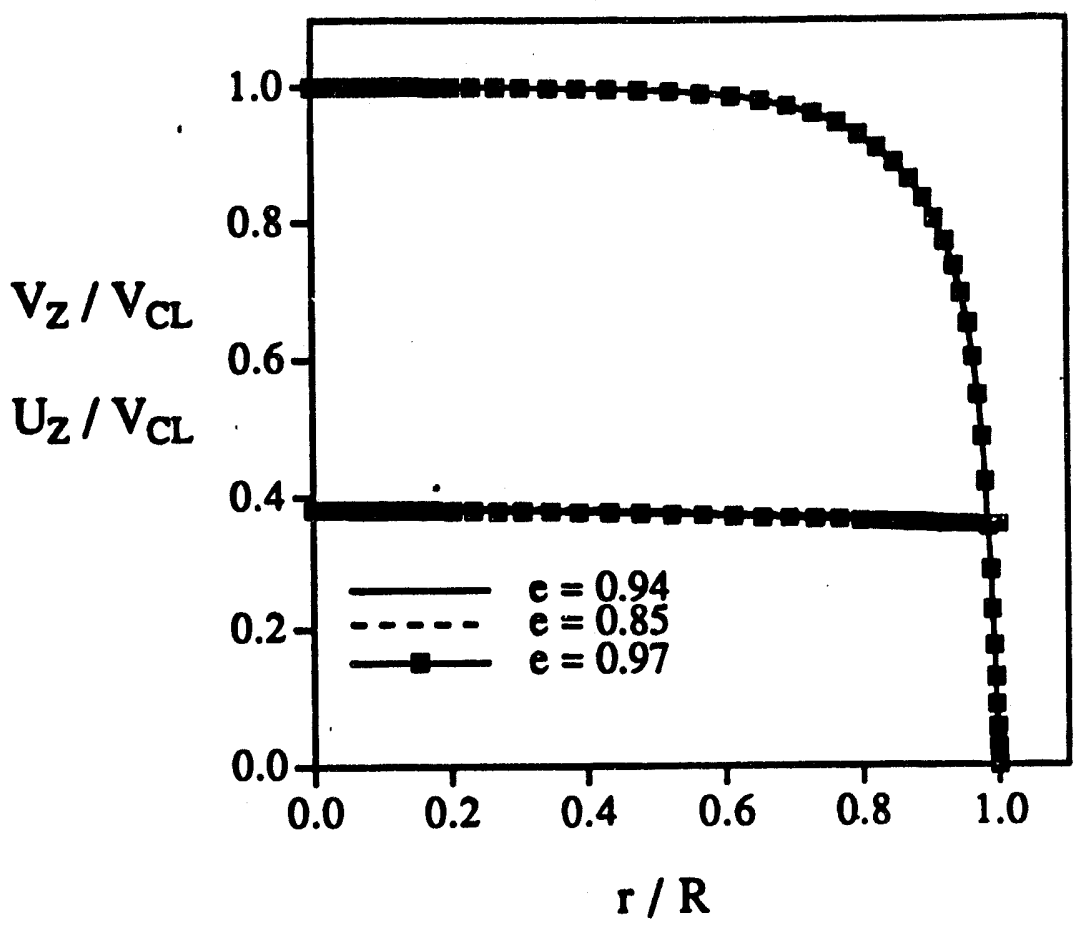

(a)

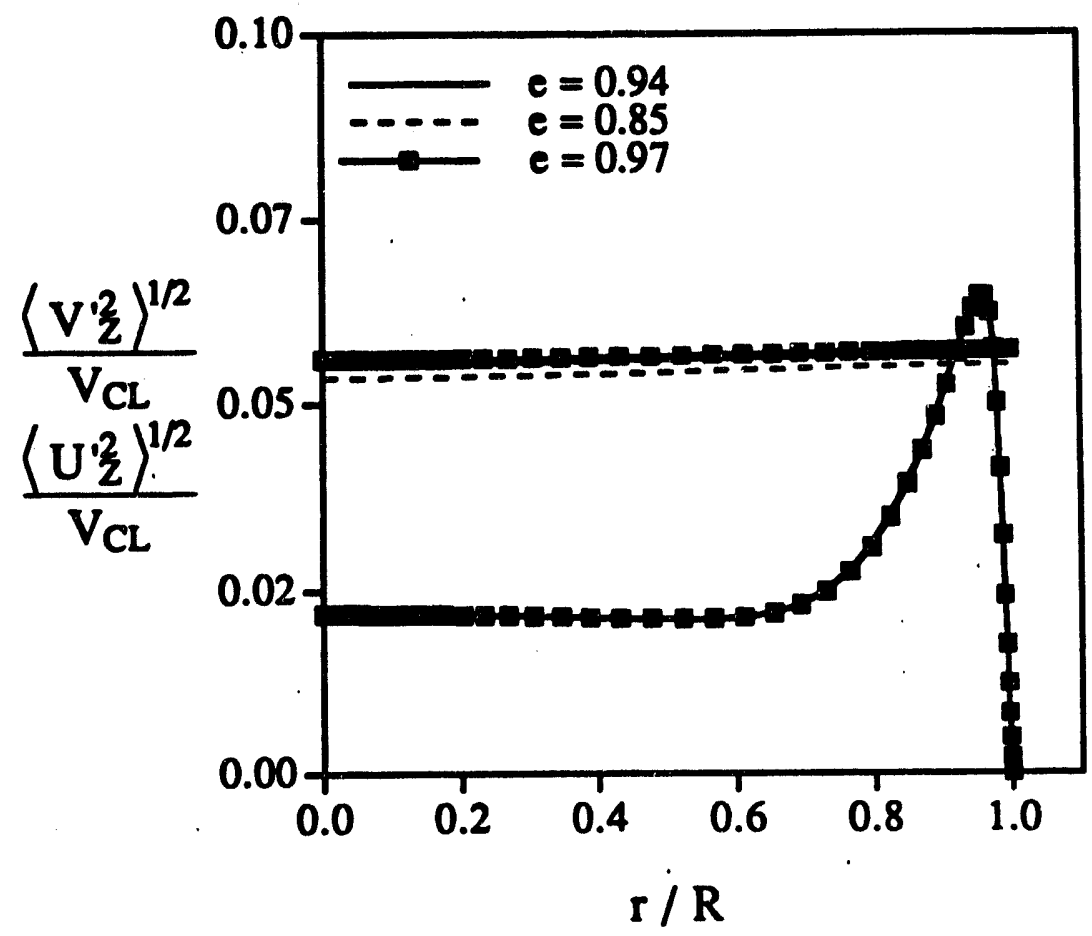

(b) 


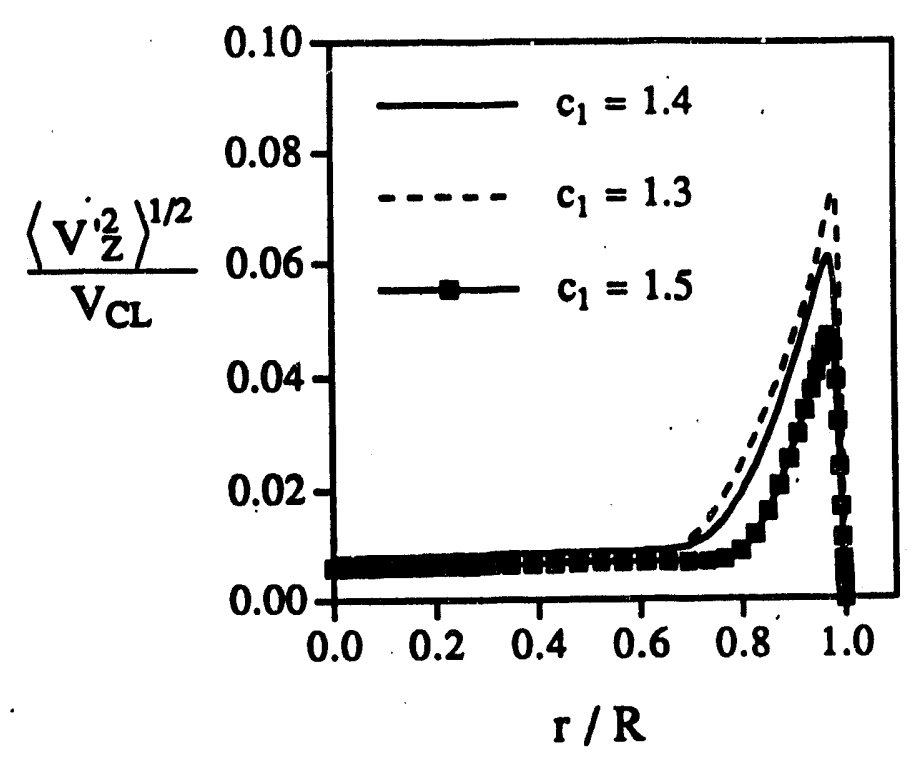

(a)

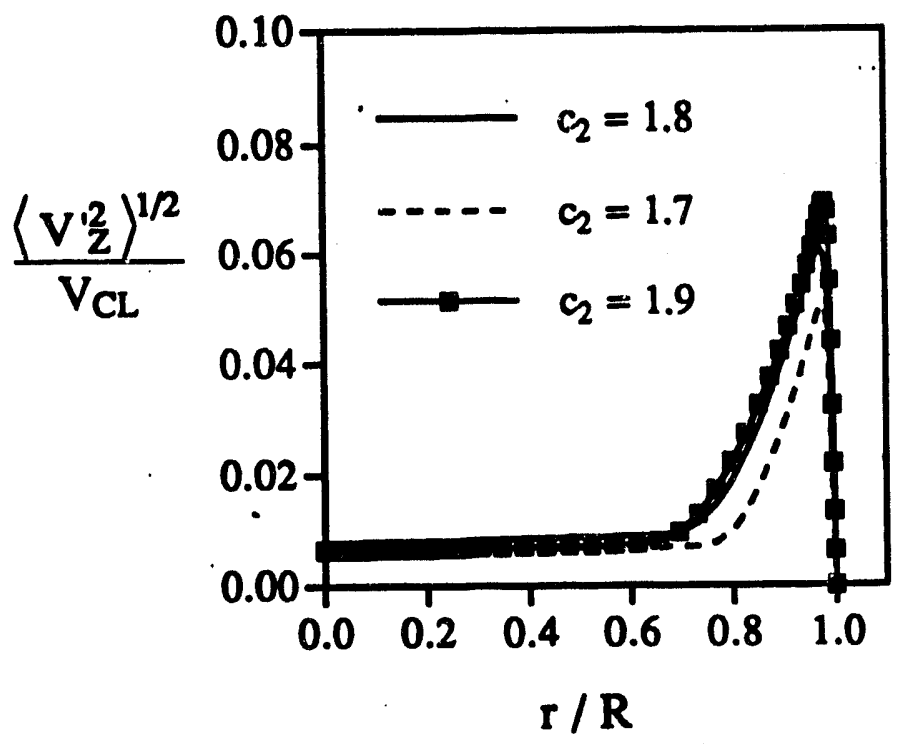

(b)

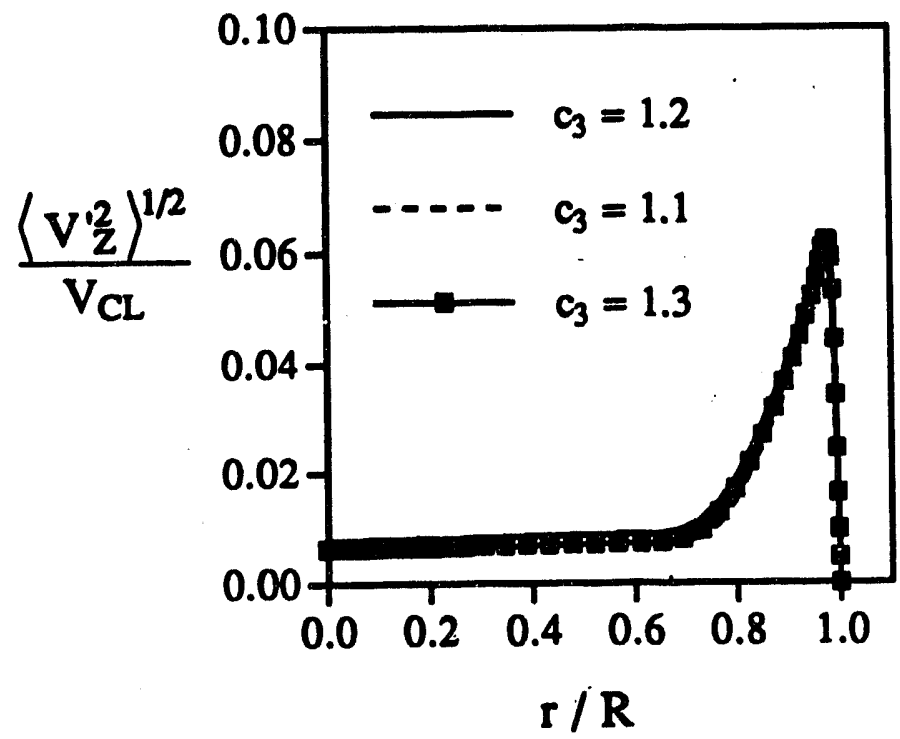

(c) 


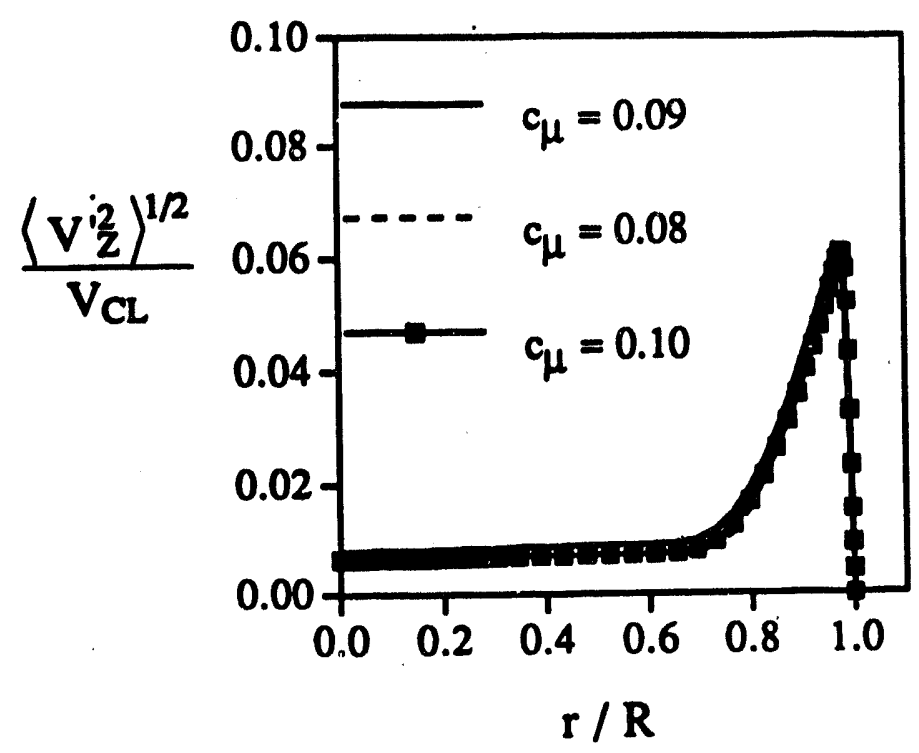

(a)

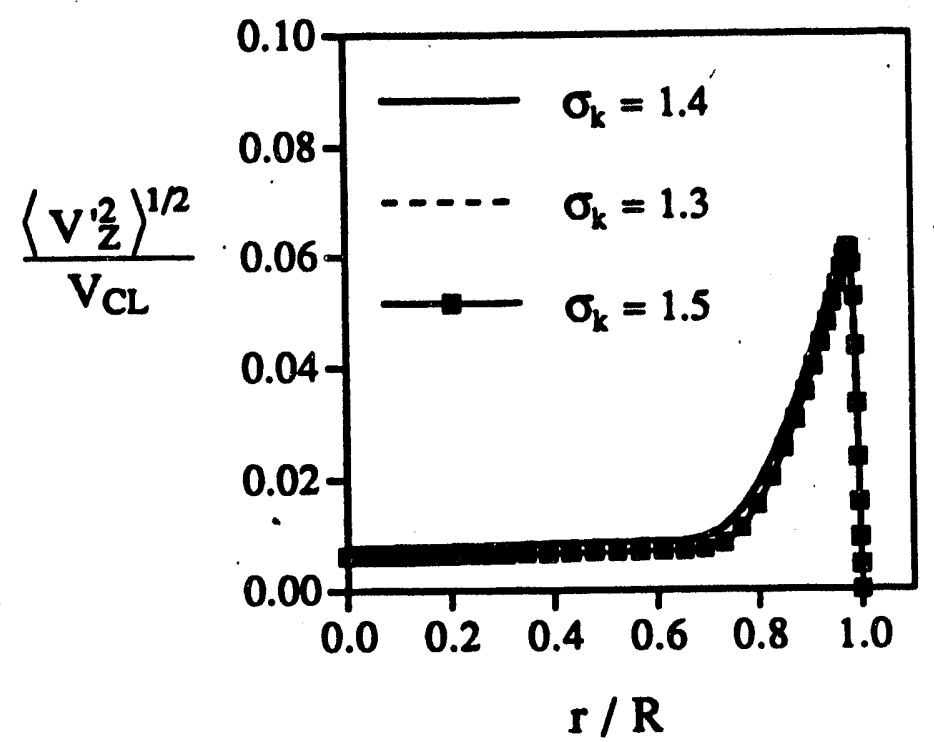

(b)

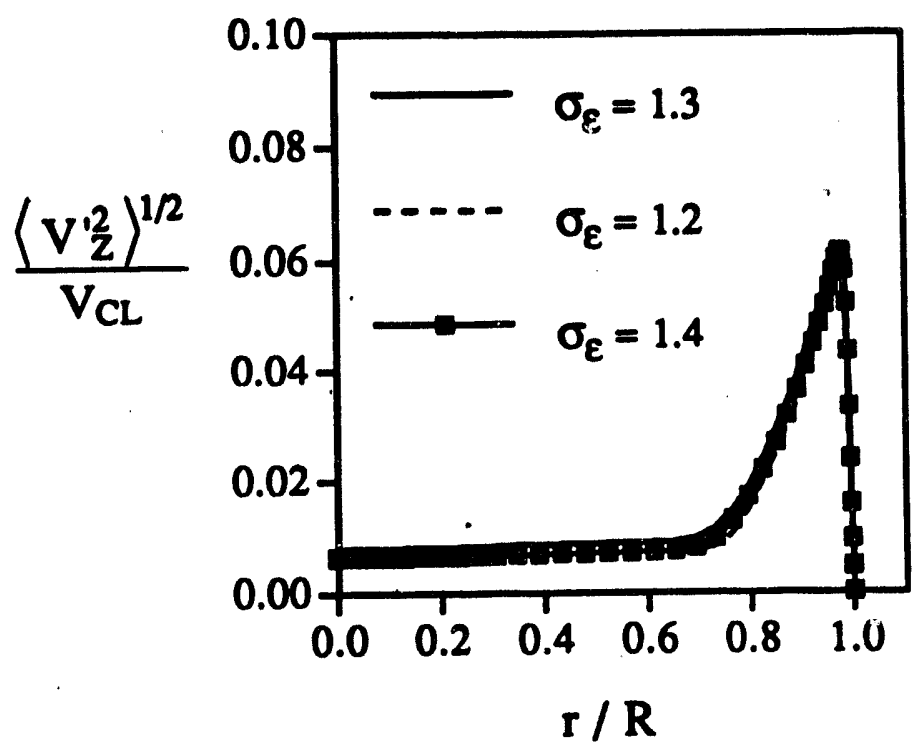

(c) 

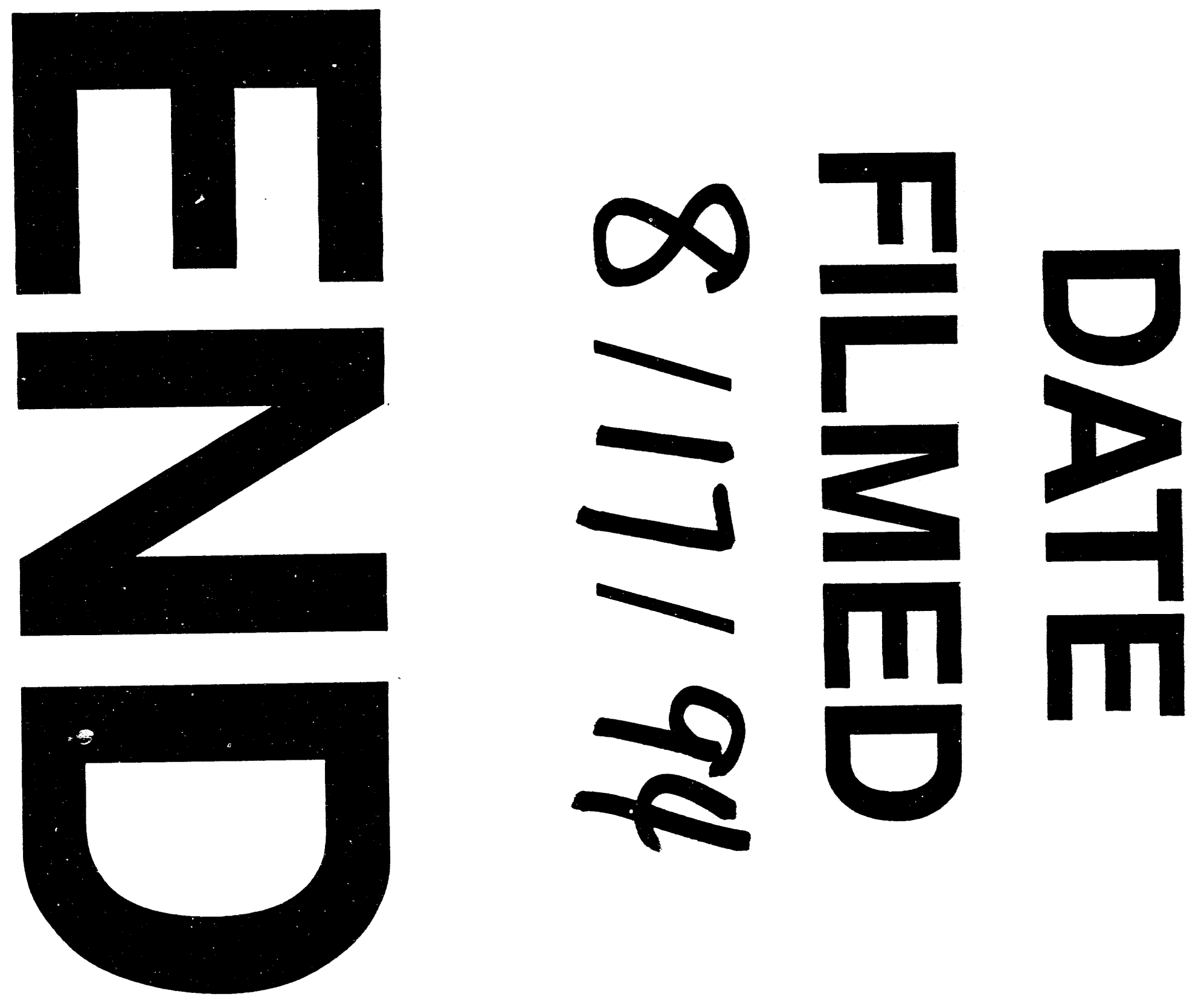


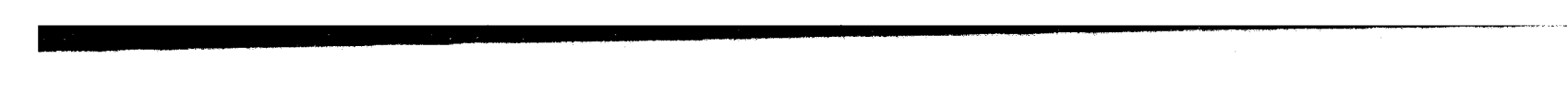

